\title{
Annotationes Epigraphicae XI \\ Zu einigen Inschriften aus den römischen Provinzen Germania inferior und Germania superior*
}

\author{
Andreas Kakoschke
}

I.

In der Erbacherhofstraße in Mainz/Mogontiacum fand sich im Jahre 1889 eine Bauinschrift (Höhe $17 \mathrm{~cm}$ - Breite $32 \mathrm{~cm}$ - Tiefe $20 \mathrm{~cm}$ ) aus Muschelkalk, die aufgrund der genannten Legion aus der Zeit zwischen 70 und 92 n. Chr. stammt (Abb.1). Der Stein in Form einer Tabula ansata ist an einigen Stellen bestoßen. Die Inschrift lässt sich allerdings noch gut entziffern. KÖRBER liest den kurzen Text wie folgt: ${ }^{1}$
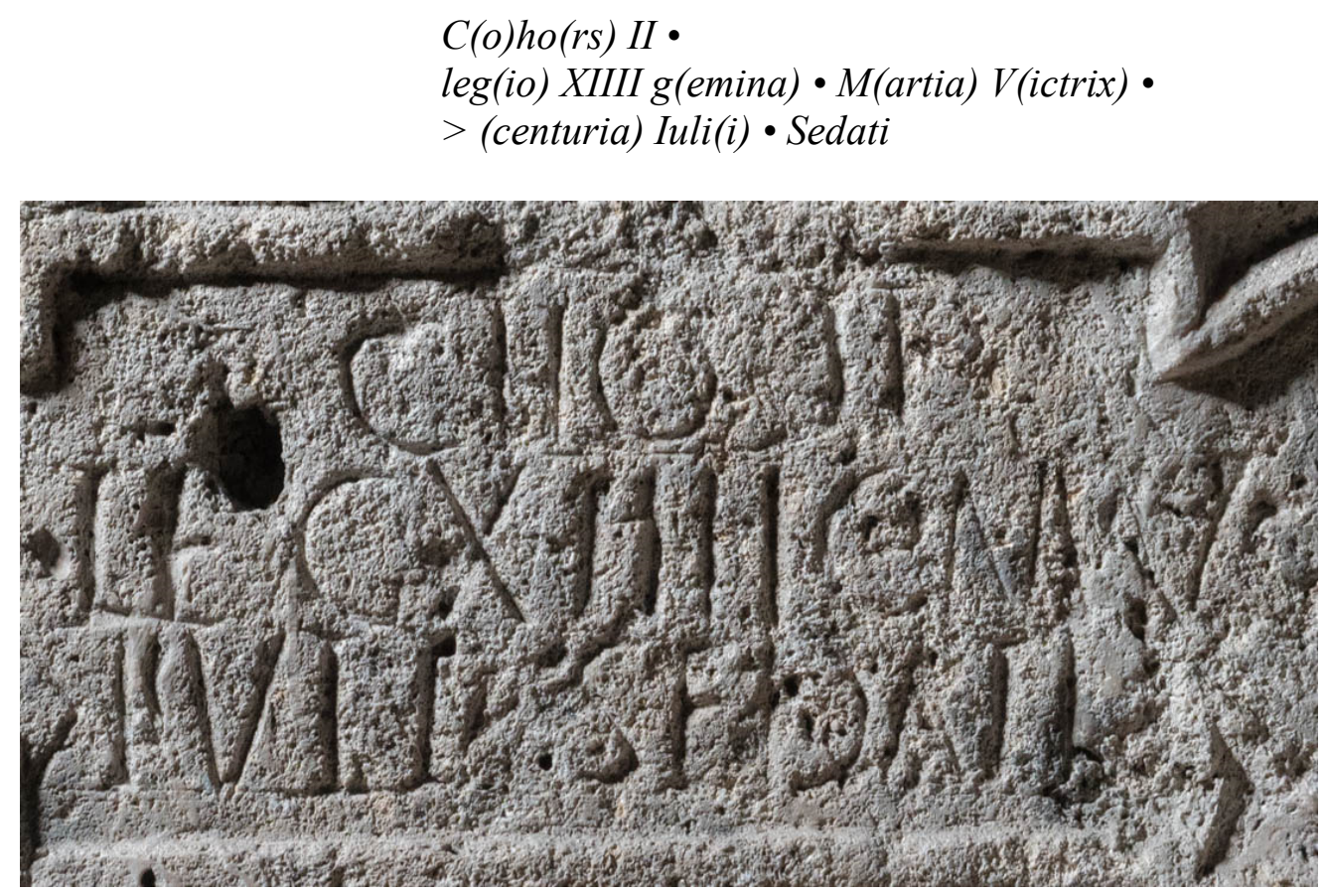

Abb.1: Bauinschrift aus Mainz/Mogontiacum: CIL XIII 6929

[C Ortolf Harl, Wien (LUPA)]

\footnotetext{
* Bei den Herausgebern der FeRA, bei Herrn Prof. Dr. K. Matijević (Flensburg) und Herrn Dr. P. Probst (Hamburg), bedanke ich mich wiederum für einige wichtige Hinweise. Für die Bereitstellung der Photos danke ich Herrn Prof. Dr. R. Wiegels und Herrn Prof. Dr. K. Matijević (CIL XIII/2-Projekt, Universitäten Trier/Flensburg) sowie Herrn Dr. O. Harl (Universität Wien). Für kritische Hinweise danke ich erneut meinem Kollegen Herrn Dr. St. Oelschig (Osnabrück). - Ligaturen bzw. Nexus werden im Folgenden durch unterstrichene Buchstaben wiedergegeben. - Die bisherigen Annotationes Epigraphicae erschienen in den vorhergehenden Ausgaben der FeRA. Die Annotationes Epigraphicae III finden sich im GFA 19 (2015), 179-197.

1 KÖRBER 1900, 36. Nr.40 (mit Zeichnung). In der Umschrift wurden die Worttrenner gemäß der Zeichnung KöRBERs hinzugefügt. - Vgl. auch die vorangegangenen Lesungen: W. VELKE, KWDZ 8 (1889), 244. Nr.1, AE 1890, 23. - Der Stein befindet sich heute im Landesmuseum in Mainz (Inv.-Nr. S 564).
} 
Im Kommentar schreibt KÖRBER: „Z. 3 hinter dem ersten I ein gebogener Strich: wohl ein Versehen des Steinmetzen." Diese Auffassung teilen offensichtlich alle folgenden Bearbeiter der Inschrift, da sie das Gentiliz des Zenturios ebenso wie KÖRBER mit dem gängigen Iulius wiedergeben. ${ }^{2}$ Akzeptiert man jedoch die eingeschlagenen Hasten als korrekte Beschriftung, muss am Beginn von Zeile 3 das eher seltene Gentiliz Fulius entziffert werden. ${ }^{3}$ Die Vertikale und die folgende kürzere, leicht gebogene Haste stehen in der kursiven Schrift schließlich eindeutig für ein F. ${ }^{4}$ Da ein kursiver Buchstabe in einer Steininschrift keinesfalls außergewöhnlich ist, ${ }^{5}$ lässt sich die Lesung Fuli $(i)$ also durchaus rechtfertigen. Nicht zuletzt weist in der vorliegenden Inschrift auch das A in SEDATI mit einer kleinen senkrechten Haste zwischen den größeren diagonalen Hasten ein kursives Merkmal auf.

Insgesamt sind nur acht Belege für das Gentiliz Fulius bekannt. ${ }^{6}$ Fast alle Nachweise für den Namen stammen aus Italien. In Spoleto/Spoletum (regio VI) finden sich gleich vier Inschriften, die den Namen in der Form Fulius bzw. Fulia, Fúlius oder Fuulius nennen. ${ }^{7}$ Ein weiterer Nachweis für die Variante Fullius lässt sich in Teano/Teanum Sidicinum (regio I) ausmachen. ${ }^{8}$ In Rom nennt eine Grabinschrift eine Frau namens Fulia Quarta. ${ }^{9}$ Und in Ostia liest man auf einer fragmentarischen Grabinschrift den Namen Fulius. ${ }^{10}$ Etwas unsicher ist dagegen ein Beleg für Full [ius] aus Potenza/Potentia (regio III). ${ }^{11}$ Schließlich tritt ein weiterer Beleg für Fullius in dem in Pannonien bzw. Italien (regio X) gelegenen Ljubljana/Emona auf. ${ }^{12}$

Angesichts der Verteilung des Namens geht man sicher nicht fehl in der Annahme, dass es sich bei dem Zenturio Fulius Sedatus um eine Person aus Italien handelt. Gegen diese Annahme spricht auch nicht das Cognomen des Mannes, der lateinische (oder keltische) Name Sedatus. ${ }^{13}$

\section{II.}

Für eine fehlerhaft eingeschlagene Weihinschrift aus Frankfurt-Heddernheim/Nida, ${ }^{14}$ die einen bis zur Unverständlichkeit verdrehten Text bietet, wurde vom Autor vor kurzem eine neue Deutung vorgeschlagen. Demnach soll die offensichtlich nicht korrekte Reihenfolge der eingeschlagenen Zeilen auf eine für den Steinmetz (bzw.

\footnotetext{
${ }^{2}$ CIL XIII 6929 („3 sic pro IVLI“), RIESE 1914, 108. Nr.920-5, SELZER 1988, 254. Nr.296 (mit Photo), RiCHIER 2004, 243. Nr.154, KAKOSCHKE 2006, 223. GN 621-447, KAKOSCHKE 2008, 309. CN 2761-2, LUPA Nr.27192, EDCS Nr.11000984, EDH Nr.HD056146.

${ }^{3}$ S. auch schon ALBRECHT 1938, 80.

${ }^{4}$ Vgl. CAGNAT $1914,8,14-15$, ReUTER/SCHOlz 2004, 18. - Ein vergleichbares F bieten u.a. auch die Grabinschriften CIL XIII 5641 (Selongey) und 5995 (= LUPA Nr.27930) (Saverne/Tres Tabernae) sowie die eingeritzte Weihinschrift CIL XIII 8546 (Neuss/Novaesium). Vgl. auch CIL XIII/5 171 (Index). Auf einem silbernen Votivblech aus Frankfurt-Heddernheim/Nida mit eingepunzter Weihinschrift [CIL XIII 7342b (= EDH Nr.HD031740)] ist dagegen entgegen den Angaben im CIL wohl kein kursives F zu lesen.

${ }^{5}$ Für Mainz/Mogontiacum vgl. z.B. die Bauinschrift CIL XIII 6934 (mit zwei kursiven L, bestehend aus einer Senkrechten und einer kleineren, leicht abgesetzten Diagonalen) sowie die Grabinschriften 1. N. 70-71. Nr.214 [mit einem kursiven bzw. keltischen E (CALIIDIVS = Caledius)] und CIL XIII 6958 (SIIX CARIIIVS SIIX F VOL FLORVS $=$ Sex. Careius Sex. f. Florus).

${ }^{6}$ Zum Namen s. Solin/SALOMIES ${ }^{2}$ 1994, 83, SCHUlZE 1904, 168, 424.

${ }^{7}$ CIL XI 4771 (= EDR Nr.128265), 4802 (= EDR Nr.164166), 4803, 4895.

${ }^{8}$ CIL X 4834 (= EDR Nr.120612).

${ }^{9}$ CIL VI 4760 (= EDR Nr.125491).

${ }^{10}$ CALDELli 2018, Nr.477.

${ }^{11}$ AE 1974, 297 (= EDR Nr.075812).

12 CIL III 13399.

${ }^{13}$ Zum Namen s. OPEL IV 61-62, DelamarRe 2007, 163, KAKOSCHKE 2008, 309-310. CN 2761.

14 1. N. 208. Nr.354 (= AE 2015, 1018 = EDH Nr.HD024436).
}

http://www.fera-journal.eu 
den Ordinator) zweideutige (handgeschriebene) Vorlage des Auftraggebers verweisen. Die Vorlage, die sicherlich in ein Wachstäfelchen geritzt war, könnte möglicherweise wie folgt ausgesehen haben:
I $\mathrm{O} \quad \mathrm{M}$
ET IVNONI REGINE
C IVLIVS
COSSVS V S L L M

Statt zweier Zeilen erkannte der wohl schreibunkundige Steinmetz drei Spalten, die er nacheinander von oben nach unten auf den Stein übertrug und so zu einem fehlerhaften (nicht korrigierbaren) Ergebnis gelangte. ${ }^{15}$

Ein vergleichbares Erklärungsmuster bietet sich vielleicht für die Deutung weiterer auffälliger Inschriftentexte aus den germanischen Provinzen an. So sei hier auf eine Weihinschrift aus Bonn/Bonna (Abb.2) hingewiesen, die sich im Jahre 1947 im Fundament der Münsterkirche fand. Der Nemesis Diana geweihte Altar (Höhe 80 $\mathrm{cm}$ - Breite $57 \mathrm{~cm}$ - Tiefe 23,5 cm), welcher angesichts der Legionsangabe aus dem frühen 2. Jahrhundert stammt, ${ }^{16}$ besteht aus Trachyt. NESSELHAUF gibt den gut zu lesenden Text wie folgt wieder: ${ }^{17}$
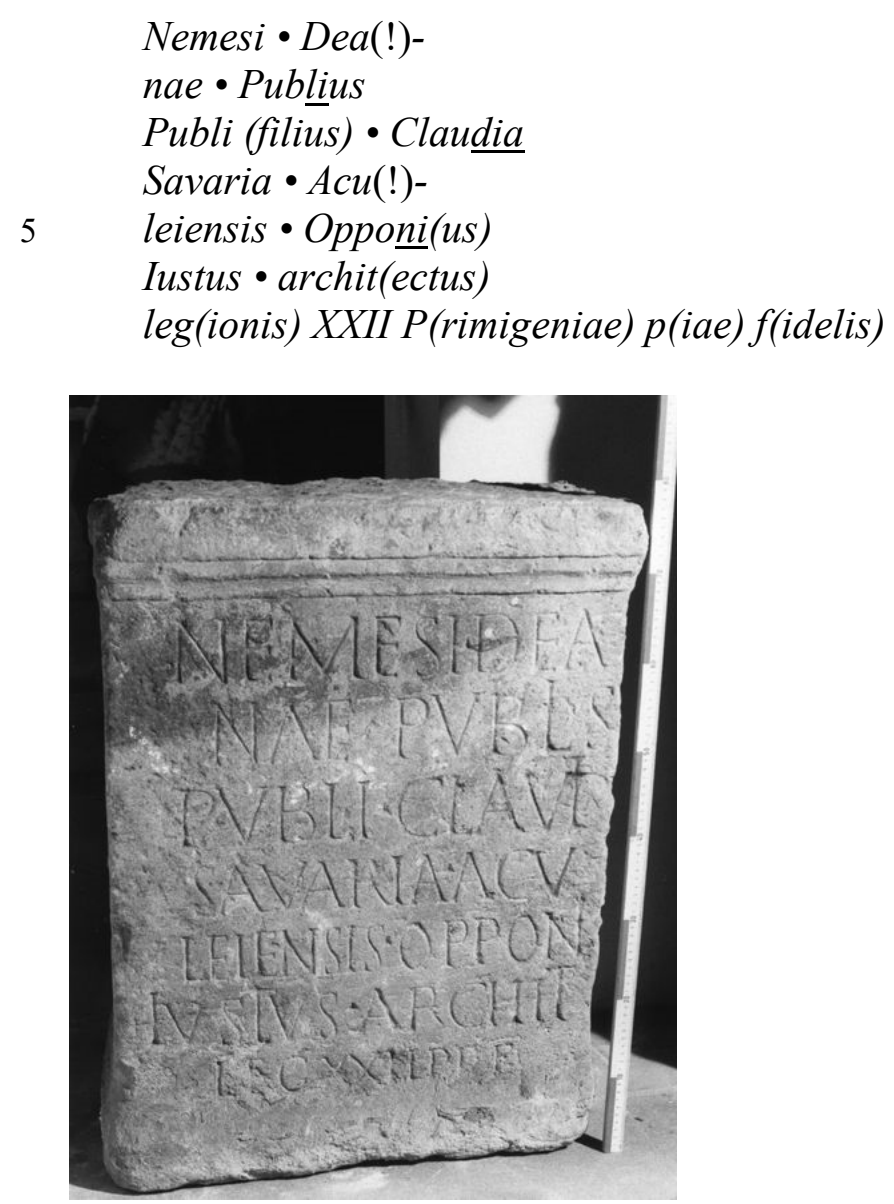

Abb.2: Weihinschrift aus Bonn/Bonna: 3. N. Nr.201

(C) CIL XIII/2-Projekt, Universitäten Trier/Flensburg)

\footnotetext{
15 A. KAKOschKe, FeRA 27 (2015), 20-21. Nr.1. Abb.1 (Photo).

${ }^{16}$ Zur Datierung s. F. OELMANN, BJb 149 (1949), 334-335. Taf.24/1 (Photo), Schwertheim 1974, $37-$ 38. Nr.39.

17 3. N. 196-197. Nr.201. Worttrenner und Ligaturen sind hier ergänzend hinzugefügt. - Der Stein lagert heute im Rheinischen Landesmuseum in Bonn (Inv.-Nr.D 1126).
} 
Der Dedikant der Inschrift ist ferner durch eine Grabinschrift aus Bonn/Bonna für einen Mann namens Iulius Paternus, einen miles der legio XXII Primigenia, bekannt. Auf dem Grabstein bezeichnet er sich ebenfalls als archit(ectus), gibt seinen Namen jedoch nur mit Opponius Iustus an. ${ }^{18}$ Die auf dem vorliegenden Stein gegebene (vollständige) Namensform, Publius Publi (filius) Claudia Savaria Aculeiensis Opponi(us) Iustus, ist äußerst bemerkenswert. Laut FORNI wäre folgende Nomenklatur zu erwarten: P. Opponius P. f. Cla. Iustus (domo) Savaria (natus oder natione) Aquileiensis. ${ }^{19}$ Für die eingeschlagene Anordnung der Namenselemente finden sich offenbar keine überzeugenden Parallelen. ${ }^{20}$ Aus diesem Grunde las PANCIERA - im Gegensatz zum Ersteditor OELMANN ${ }^{21}$ und dem zuvor zitierten NESSELHAUF - auf dem Stein nicht einen, sondern zwei Namen. PANCIERA zufolge wurde die Inschrift von einem Publius Publi(cius oder -lius) Aculeiensis und von dem architectus Opponius Iustus dediziert. ${ }^{22}$ Es ist aber unwahrscheinlich, dass in der Inschrift ein Dedikant mit ausgeschriebenem Pränomen, ausgeschriebener Tribusangabe und zweifacher Herkunftsangabe, der andere nur mit Gentiliz und Cognomen sowie der Angabe architectus legionis genannt wird. ${ }^{23}$

Geht man weiterhin von der Nennung eines einzigen Dedikanten aus, bietet sich als Erklärung für die ungewöhnliche Anordung der Namenselemente - abgesehen von einer nicht regelgerechten, nicht üblichen oder originellen Nomenklatur (für die sich irgendwann möglicherweise noch weitere Belege finden) - vielleicht auch ein Steinmetzfehler an. Die Vorlage für den Steinmetz könnte dabei wie folgt ausgesehen haben:

\section{NEMESI DEANAE PVBLIVS PVBLI CLAVDIA SAVARIA ACVLEIENSIS}

\section{OPPONIVS IVSTVS ARCHIT LEG XXII P P F}

Wie dem zuvor erwähnten Steinmetz aus Frankfurt-Heddernheim/Nida mag auch dem Steinmetz aus Bonn/Bonna bei der Übertragung des Textes auf den Stein ein Fehler unterlaufen sein. Dabei erkannte er nicht zwei Zeilen, sondern zwei Spalten, deren Worte er nacheinander von oben nach unten las und entsprechend (mit einer selbst gewählten Zeilenaufteilung) auf den Stein übertrug.

Es bleibt zu erwähnen, dass die Namensform Publius Opponius Iustus Publi (filius) Claudia Savaria Aculeiensis mit ihrer nachgestellten (ausgeschriebenen) Filiation gleichfalls ungewöhnlich ist. Für diese Namensform lassen sich jedoch vereinzelte Beispiele angeben. So führte (in der ersten Hälfte des ersten Jahrhunderts n. Chr.) ein italischer Legionär aus Strasbourg/Argentorate den Namen Ti[b]erius Babuleius Garullus Tib(e)ri (filius) O(u)fent(ina) Medioleanio(!). ${ }^{24}$

\footnotetext{
${ }^{18}$ CIL XIII 8082 [= LEHNER 1918, 245-246. Nr.630 = LUPA Nr.15544 (mit Photo)].

${ }^{19}$ FORNI 1985, 116. Anm.10.

${ }^{20}$ FORNI 1985, 116. Anm.10 verweist am Rande auf einen Tib(erius) Tiberi f(ilius) Cla(udia) Satto Camboduno [CIL III 15162 (= EDH Nr.060423)] aus Budapest/Aquincum. Jedoch wurde laut FORNI hier in der Nomenklatur zwischen Pränomen und Filiation sicher das Gentiliz unterschlagen.

${ }^{21}$ F. OELMANN, BJb 149 (1949), 334-335. Taf.24/1 (Photo) (= AE 1953, 93).

22 S. PANCIERA, Epigraphica 19 (1957), 3-9 (mit Photo) (= AE 1960, 160). Vgl. auch die entsprechenden Angaben in der EDH (Nr.HD018811). Der Lesung PANCIERAs entsprechend führt auch das OPEL I ${ }^{2} 70$ Aculeiensis unter den Belegen für das Cognomen Aquileiensis auf. S. noch SPICKERMANN 2008, 120. Nr.10 der ebenfalls zwei Dedikanten angibt: Publius Publi (filius) Claudia (tribu) Savaria und Aculeiensis Opponi(us) Iustus.

${ }^{23}$ FORNI 1985, 116-117. Anm.10.

${ }^{24}$ CIL XIII 5976 (= RICHIER 2004, 168-169. Nr.27). - S. ferner u.a. folgende vergleichbare Personennamen aus den germanischen Provinzen: M(arcus) Vibius Maurinus M(arci) f(ilius) domo Papiria Augusta [AE 1974, 451 (= IKöln² 267-268. Nr.314), Köln/CCAA], T(itus) Iulius Tuttius T(iti) f(ilius)
}

http://www.fera-journal.eu 
III.

In Eschweiler-Fronhoven (Städteregion Aachen) entdeckte man im Jahre 1980 im Vorfeld des Braunkohletageabbaus Zukunft-West ein Matronen-Heiligtum und zahlreiche Weihinschriften. Die Steine aus der Zeit zwischen 150 und 225 n. Chr. sind soweit erkennbar - den Matronae Alaferhuiae und Amfratninehae geweiht. ${ }^{25}$ Unter den Stücken befindet sich ein in zwei Teile zerbrochener fragmentarischer Weihaltar (Höhe $38 \mathrm{~cm}$ - Breite 32,5 cm - Tiefe $16 \mathrm{~cm}$ ) aus graugelbem Sandstein (Abb.3), auf dessen Seitenflächen noch ein Baum (links) bzw. ein Blattarrangement (rechts) zu erkennen ist. Der Ersteditor der Inschrift, RÜGER, gibt den Text dieses Steins in Majuskelschrift und Umschrift wie folgt wieder: ${ }^{26}$

$$
\begin{aligned}
& \text { [---] } \\
& \text { ?] ALETIAN[I?]VS[..]IC[E?] } \\
& \text { ?]R • ET • IANVARIA • ALB } \\
& \mathrm{VCI} \cdot \mathrm{F} \bullet \mathrm{ET} \cdot \mathrm{NEGALATI} \\
& \text { ANI } \bullet \text { ACCEPTVS } \cdot \underline{E T} \\
& 5 \text { CERTVS } \bullet \text { SIMILIS } \\
& \mathrm{F} \cdot \mathrm{L} \cdot \mathrm{M}
\end{aligned}
$$

[Matronis ---] / [.] Aletian[i] us .. IC[E]/[.]R et Ianuaria Alb/uci f(ilia) et Negalati/ani Acceptus et $\hat{\rho}$ Certus Similis / f(ilii) l(ibentes) m(erito)

Laut RÜGER bezeugt die Inschrift zwei neue Gentilnomina, Aletianius (Zeile 1) und Negalatianius (Zeile 3/4). Wie RÜGER sehen die meisten Epigraphiker oder Onomastiker in dem im Plural genannten Namen NEGALATIANI einen Beleg für den Namen Negalatianius. ${ }^{27}$ Allerdings kann der Name im Singular ebenso Negalatianus gelautet haben. In dieser Form gibt offenbar nur DELAMARRE den Namen an. ${ }^{28}$ Entspechend muss das Gentiliz in der ersten erhaltenen Zeile nicht Aletianius oder [--Jaletianius gelautet haben. Schließlich ist die von RÜGER mit einem Fragezeichen verschriftlichte - aber in den Textwiedergaben nicht durch diakritische Zeichen angegebene - NI-Ligatur aufgrund des fragmentarischen Zustands des Steins nur eine Annahme. Der Name kann daher ebenso als Aletianus bzw. [---]aletianus gelesen werden. Da es sich bei den Dedikanten wohl um Verwandte handelt, spricht alles für eine Ergänzung des Namens zu [Neg]aletianus. ${ }^{29}$ Es läge dann wahrscheinlich eine

Claudia Viruno [CIL XIII 8289 (= IKöln² 277. Nr.327), Köln/CCAA), L(ucius) Pollenti(us) Dexter L(uci) fil(ius) domo Save(ria)(!) [AE 2012, 989 (= EDH Nr.HD072686), Avenches/Aventicum), Q(uintus) Bisius Secund(us) Q(uinti) f(ilius) domo Brixsa(!) (CIL XIII 8733, Nijmegen/Noviomagus).

${ }^{25}$ RÜGER 1983, 115-158. Nr.4-42.

${ }^{26}$ RÜGER 1983, 154-156. Nr.39. Abb.39 (Photo). - Der Weihestein befindet sich heute im Rheinischen Landesmuseum in Bonn (Inv.-Nr.80.3313).

${ }^{27}$ B. BEYER, in: RÜGER 1983, 163 (Index zu den Inschriftenfunden 1975-1982 aus dem Rheinland), AE 1984, 693, SOLIN/SALOMIES² 1994, 126, 474, 485, SPICKERMANN 1994, 360-361, KAKOSCHKE 2006, 283. GN 845, RAEPSAET-CHARLIER 2011, 221, EDH Nr.HD001398.

${ }^{28}$ DELAMARRE 2007, 139.

${ }^{29}$ Zur Lesung des Namens vgl. auch B. BEYER, in: RÜGER 1983, 162 (Index zu den Inschriftenfunden 1975-1982 aus dem Rheinland) (,Aletian[i]us“), SPICKERMANN 1994, 360-361. Nr.3 mit Anm.271 (,Aletian[i?]us ... eine bisher nicht bezeugte einheimische Bildung aus Aletus“), Solin/SALOMIES ${ }^{2}$ 1994, 474, 485 („,[Neg]aletian[i]us(?)“), KAKOSCHKE 2006, 67. CN 48 („Möglicherweise auch [---]aletian[i]us oder [Neg]aletian[i]us“), DELAMARRE 2007, 17 („Aletianus“), BILLER 2010, 257 (,Aletian[i?]us“), EDCS Nr.08400420 (,Aletian[i]us“), EDH Nr.001398 (,[Neg?]aletian[i]us“).

http://www.fera-journal.eu 
Verschreibung des Namens Negalatianus vor, sofern es sich nicht bei dem im Plural genannten Namen NEGALATIANI um einen Steinmetzfehler handelt.

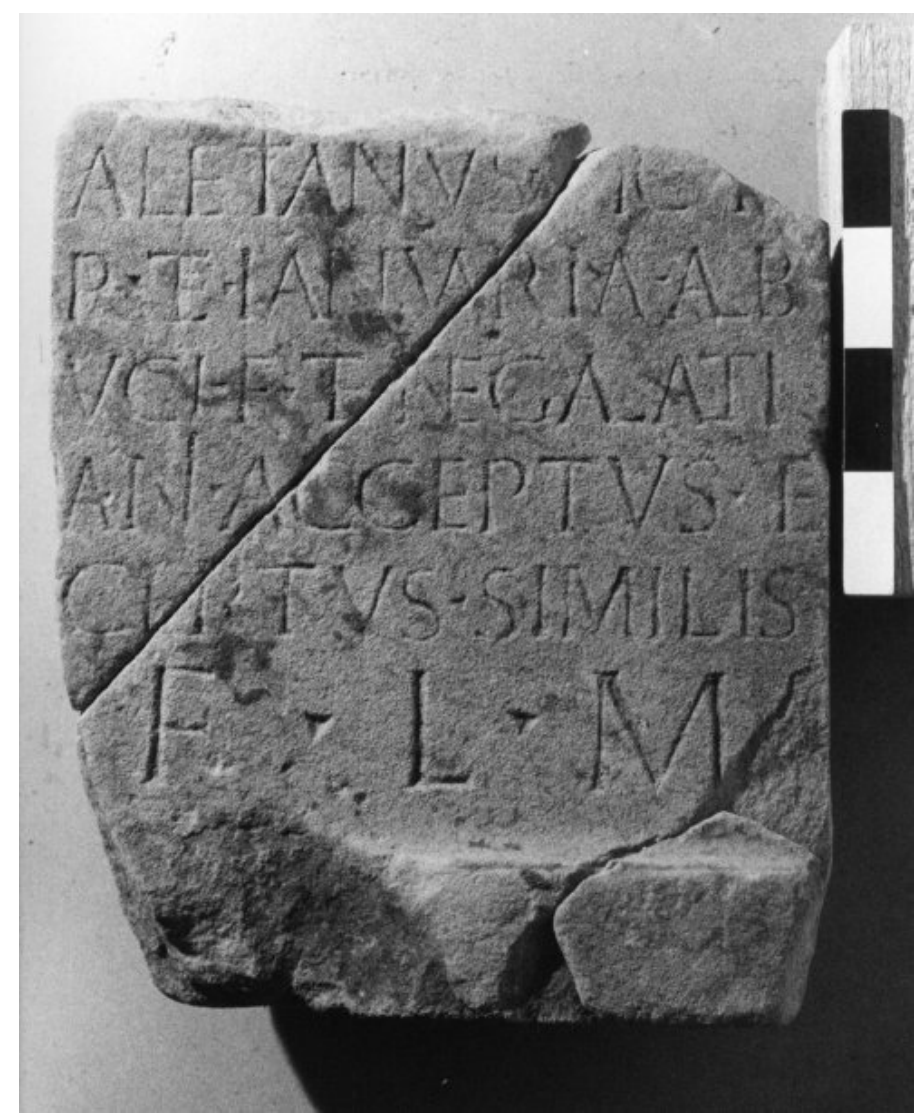

Abb.3: Fragmentarische Weihinschrift aus Eschweiler-Fronhoven: AE 1984, 693 (C) CIL XIII/2-Projekt, Universitäten Trier/Flensburg)

RÜGER verweist ergänzend auf das aus Köln-Bocklemünd/CCAA bekannte (Pseudo)Gentiliz Negalaeti(us), das sicher mit den in Eschweiler-Fronhoven bezeugten Namen verwandt ist. ${ }^{30}$ Ergänzend kann nun zudem ein Beleg für Negalet[---] aus Britannien, aus Chesterholm/Vindolanda, angeführt werden. ${ }^{31}$ Die unterschiedliche Verschriftlichung der verwandten Namen mit AE bzw. E lässt die unterschiedliche Schreibung, den mutmaßlichen Steinmetzfehler, in der Inschrift aus Eschweiler-Fronhoven vielleicht verständlicher erscheinen.

Bemerkenswert ist schließlich am Rande noch die Tatsache, dass der Name in der zuvor erwähnten Inschrift aus Köln-Bocklemünd/CCAA als NEG•ALAETI, also mit einem Silbentrenner, realisiert wurde. Das Zeichen trennt den Namen somit nach der Silbe Neg-. Entsprechend erfolgt in der Inschrift aus Eschweiler-Fronhoven der Zeilensprung offenbar nach den (verlorenen) Buchstaben NEG. Zwar vermutet RÜGER in den ersten erhaltenen Zeilen der Inschrift jeweils vor dem ersten Buchstaben noch einen weiteren, verlorenen Buchstaben (s.o.), doch fehlt hierfür eindeutig der Platz (siehe Abb.4). Zu lesen ist daher wohl [Neg]/aletianus.

Die hier vorgetragenen Einwände gegen die gängige Lesung des Namens Negalatianius lenken die Aufmerksamkeit auf weitere einheimische Gentilnomina mit der charakteristischen Endung -ianus. Fast alle Belege stammen aus dem südlichen

\footnotetext{
${ }^{30}$ CIL XIII 8513 [= IKöln ${ }^{2}$ 187-189. Nr.209 (mit Photo)].

${ }^{31}$ AE 2007, 874 (= EDH Nr.HD068772).
} 
Niedergermanien. So belegt eine Inschrift aus Morken-Harff einen Mann namens L. Laubasnianus Ammalenus. ${ }^{32}$ Eine Inschrift aus Inden-Pier nennt einen Dedikanten namens T. Tattianus [S?] eranus $^{33}$ und eine Grabinschrift aus Jülich/Iuliacum gilt einem C. Ucsperianus Vitalis. ${ }^{34}$ Neben solchen nur einmal bezeugten Namen finden sich auch mehrfach belegte -ianus-Namen wie Bassianus und Sabinianus. Alle genannten Namen treten fast nur in der Germania inferior auf. ${ }^{35}$

Bei allen zuvor erwähnten Namen handelt es sich zweifellos um einheimische Pseudogentilnomina. Noch ALFÖLDY hatte einen Namen wie Bassianus als Bassian(i)us gelesen. ${ }^{36}$ CLAUSs stellt dagegen zutreffend fest, dass es sich bei den genannten Namen um eine ,in germanisch-keltischen Gebieten übliche Eigenart“ handelt und dass eine Korrektur, etwa von Bassianus in Bassian(i)us, nicht nötig ist. ${ }^{37}$

Der äußeren Form nach gleichen die Pseudogentilnomina einem einfachen Cognomen oder Nomen simplex mit einer-ianus-Erweiterung. Wobei ein Cognomen auf-ianus natürlich auch aus einem Gentilnomen hervorgegangen sein kann. Entsprechende Cognomina mit einem Suffix -ianus haben jedenfalls stets eine patronymische Bedeutung. ${ }^{38}$ Dieser Eigenschaft verdanken die Namen im nördlichen Bereich des Imperiums wohl ihre Verwendung als Pseudogentilnomina, die bekanntermaßen ebenfalls als Patronymika aufgefasst wurden. ${ }^{39}$

Als Basis für den Namen Bassianus darf das Cognomen bzw. Nomen simplex Bassus angenommen werden. Laubasnianus wurde sicher vom Namen Laubasnius abgeleitet, Tattianus entsprechend von Tata oder Tatius, während für Sabinianus wohl nur die Basis Sabinus angenommen werden kann. Die hier ausführlicher besprochenen Namen aus Eschweiler-Fronhoven, Negalatianus bzw. Negaletianus, dürften zur Basis *Negalatus bzw. *Negalaetus führen.

Clauss bezeichnet einen Namen wie Tattianus oder Bassianus als „gentilicium-cognomen ${ }^{\text {“40 }}$ bzw. als ein ,als gentilicium verwendete(s) cognomen“. ${ }^{41}$

\footnotetext{
32 3. N. 205-206. Nr.233.

${ }^{33}$ AE 2001, 1429 (= EDH Nr.HD006747).

${ }^{34}$ CIL XIII 7877.

${ }^{35} \mathrm{Zu}$ den Nachweisen s. im Einzelnen die Tabelle 1 am Ende des Beitrages.

${ }^{36}$ ALFÖLDY 1968, 34. Nr.4, 70. S. auch BILLER 2010, 218 [,Die korrekte Form dieses patronymischen Gentilicium würde Bassianius lauten. Demzufolge ergänzt Alföldy in seiner Publikation der Inschrift auch das fehlende I im Namen."].

Entsprechende fehlerhafte Lesungen von Pseudogentilnomina auf -ianus sind häufiger zu beobachten: RIESE 1914, 354. Nr.3490 [,Aprian(i)us“], 395. Nr.4005a [„Aprian(i)a“], LEHNER 1918, 117. Nr.243 („Aprian[i]us“), 2. N. 72. Nr.78 („Das Gentile Bassiana gleich Bassiania ...“), 100. Nr.172 („Der Name der Frau ist unklar: Bassiana anstatt Bassiania als Gentilnamen kommt auch sonst vor ..."), G. AlfÖLDY, ES 4 (1967), 2. Nr.2 [„Bassian(i)a Materna ... Bass(i)an(i)a Pa(t)erna“], A. DEMAN, ANRW II.4 (1975), 307. Nr.G22a [„Bassian(i)us“], RÜGER 1983, 127 („,.. handelt es sich gewiß um die verschriebene Gentilizbildung Bassianius.“), SPICKERMANN 1994, 303 [„Bassian(i)a“], 334. Anm.139 („Die Schreibweise Bassiana für Bassiania ...“), 342. Nr.2 [„Bassian(i)a Materna ... Bass(i)an(i)a Pa(t)erna“], KAKOschKe 2002, 407-408. Nr.5.19 [,Bassian(i)a Materna ... Bass(i)an(i)a Pa(t)erna“], RAEPSAET-ChARLIER 2011, 211 [,_Laubasnian(i)us“], P. DE BERNARDo STEMPEL, BNF 49 (2014), 91 („Aprian<i>us“), LUPA Nr.16798 [,Bassian(i)a“], EDH Nr.HD014378 [„Bassian(i)us“], HD023928 [,Bassian(i)a“], HD076819 [„Bassian(i)a Materna ... Bass(i)an(i)a Pa(t)erna“], HD079523 [,Appian(i)us“].

37 CLAUSS 1976, 12. Unverständlich ist demgegenüber folgende dort vorangestellte Aussage von Clauss: „Der Dedikant (M. Bassianus Atticus, A.K.) trägt ein gentilicium, das zwar nicht die korrekte, aber die vom Dedikanten für korrekt gehaltene Form bietet.“

${ }^{38}$ KAJANTO 1965, 109-110.

${ }^{39}$ Zum patronymischen Charakter von Pseudogentilnomina s. A. KAKOsCHKE, FeRA 26 (2015), 15-16, ders. FeRA 30 (2016), 9-10 (jeweils mit weiteren Hinweisen).

${ }^{40}$ Clauss 1976, 12.

${ }^{41}$ Clauss $1976,7$.
} 
Da neben einheimischen Bildungen wie Bassianus, Tattianus oder Sabinianus auch italische Gentilnomina auf -ianus existieren, charakterisiert man die Bildungen m.E. aber besser als Pseudogentilnomina. Der äußeren Form nach gleichen einige dieser Namen nämlich gut italischen Namen, wie Lusianus, Marcianus oder Pupianus, die auch für ortsfremde Personen in den germanischen Provinzen bezeugt sind. ${ }^{42}$ Oder anders formuliert: Ebenso wie die gängigeren Pseudogentilnomina auf -ius oder -inius imitieren die selteneren -ianus-Namen zumindest hinsichtlich der Endung italische Geschlechternamen. Die Bildungsweise der Gentilnomina, Ableitung vom Namen des Vaters, beruht jedoch auf einheimischen Traditionen. War die Basis für einen -ianus-Namen dabei nicht ein lateinischer Name, sondern ein einheimischer bzw. germanischer Name, wie etwa Laubasnius, *Negalatus oder Uxperus, sind/waren die Namen selbstverständlich gleich als einheimische Bildungen zu erkennen. ${ }^{43}$

Zurück zur Inschrift aus Eschweiler-Fronhoven: Die Lesung der Inschrift lässt sich möglicherweise in weiteren Punkten modifizieren. Welcher Matronengruppe der Stein geweiht war, den Alaferhuiae oder den Amfratninehae, bleibt unbekannt. Da sich der Name des ersten Dedikanten über zwei Zeilen zog, darf man aufgrund der Länge der Matronenbeinamen vermuten, dass über der noch vorhandenen Zeile eher zwei Zeilen verlorengingen. Dass am Anfang der Inschrift MATRONIS stand (vgl. Abb.4) ist jedoch nicht sicher, da die Bezeichnung auf vielen Steinen aus EschweilerFronhoven fehlt. ${ }^{44}$ Ferner lässt sich das Cognomen des ersten Dedikanten vielleicht zum gängigen Victor, eventuell zum eher seltenen Pictor ergänzen. ${ }^{45}$ Allerdings kann dann am Ende der Zeile aufgrund der Platzverhältnisse nur ein kleineres O oder eine (eher ungewöhnliche) TO-Ligatur gestanden haben.

Bei den Dedikanten der Weihung handelt es sich höchstwahrscheinlich um eine Familie - Vater, Mutter und drei Söhne. ${ }^{46}$ Daher ist RÜGERs Auflösung der drei Buchstaben in der letzten Zeile zu f(ilii) l(ibentes) m(erito) fraglich, da der Stein sicher nicht nur von den Söhnen dediziert wurde. ${ }^{47}$ Die größer eingeschlagenen (zusammengehörigen) Buchstaben FLM stehen sicherlich für eine Schlusswendung und somit m.E. eher für f(ecerunt) l(ibentes) $m$ (erito). Zwar ist die Schlussformel nicht allzu gängig, aber sie findet sich in dieser und in leicht abgewandelter Form auch in den germanischen Provinzen. ${ }^{48}$

Zusammenfassend darf man somit folgende Neulesung des Textes vorschlagen:

\footnotetext{
${ }^{42} \mathrm{Zu}$ den Namen s. KAKOSCHKE 2006, 247. CN 696, 258-259. CN 742, 326. CN 1018 (jeweils mit Belegen).

${ }^{43}$ Zur jeweiligen Basis der -ianus-Namen s. die Tabelle 1 am Ende des Beitrages.

${ }^{44}$ In der Rekonstruktionszeichnung (Abb.4) wurde mit ALAFERHVIS [vgl. RÜGER 1983, 123-124. Nr.11 (= AE 1984, 674)] beispielhaft die kürzeste in Eschweiler-Fronhoven bezeugte Variante eines Matronenbeinamens eingesetzt.

${ }^{45} \mathrm{Zu}$ Pictor s. KAKOSCHKe 2008, 199. CN 2369. Zu Victor s. KAKOSCHKE 2008, 452-454. CN 3321. Eine Ergänzung zu Victor wird bereits in der AE, im Kommentar zu AE 1984, 693, erwogen.

${ }^{46}$ Vgl. dagegen SPICKERMANN 1994, 360-361. Nr.3 („Die Stifter waren drei Männer und eine Frau. Es könnten zwei Geschwisterpaare genannt sein, wobei Aletian[i]us, dessen Cognomen zerstört ist, und Ianuaria Kinder des Albucius waren, während sich die beiden Negatilanii (sic) als Söhn des Similis bezeichnen.").

${ }^{47}$ RÜGER 1983, 156 erwägt auch die Lesung $f$ (ratres) l(ibentes) m(erito).

48 Verwiesen sei u.a. auf CIL XIII 5198 (Windisch/Vindonissa): feci[t] libe(n)s merito, 6031 (Gundershoffen): v(otum) f(ecit) l(ibens) l(aetus) m(erito), 7272 (Wiesbaden/Aquae Mattiacorum): fecerun [t] l(ibentes) l(aeti) m(erito), 11678a (Schweighouse-sur-Moder): f(ecit) l(ibens) [l(aetus) m(erito)], AE 2005, 1121 (Osterburken): ex voto fecit l(ibens) [l(aetus) m(erito)]. S. ferner u.a. RIB 151 (Bath/Aquae Sulis): f(ecit) l(ibens) m(erito), 1537 (Carawburgh/Brocolitia): fecit lib(ens) mer(ito).
}

http://www.fera-journal.eu 

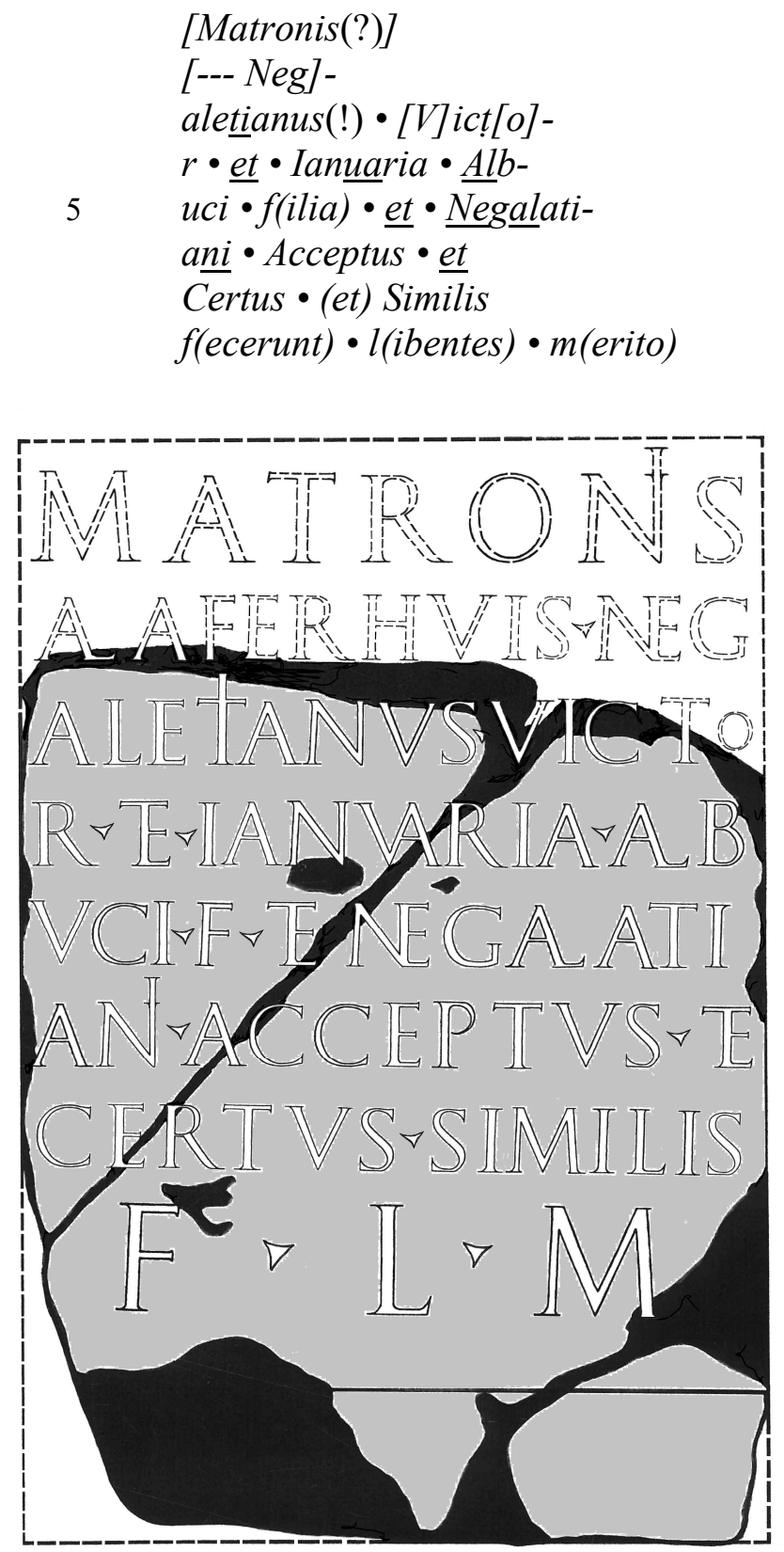

Abb.4: Leicht idealisierte Rekonstruktionszeichnung der Weihinschrift AE 1984, 693 aus Eschweiler-Fronhoven mit teilweise beispielhaften Ergänzungen (Zeichnung A.K.)

Die vorangegangenen Bemerkungen hinsichtlich der Lesung und onomastischen Einordung des überlieferten Gentilnomens aus Eschweiler-Fronhoven führen zu einer teilweisen Neubewertung einiger überlieferter Namen aus den germanischen Provinzen. Einzelne Fälle sollen an dieser Stelle ausführlicher besprochen werden.

IV.

Im Zuge der Ausgrabungen im Matronenheiligtum von Nettersheim-Pesch (Kr. Euskirchen) zu Beginn des 20. Jahrhunderts fand sich im Jahr 1913 (im Hof A) ein fragmentarisch erhaltener Weihealtar (Höhe $49 \mathrm{~cm}$ - Breite $33 \mathrm{~cm}$ - Tiefe $15 \mathrm{~cm}$ ) aus rotem Sandstein (Abb.5). Der unvollständige Text aus der Zeit zwischen 150 und 250 n. Chr. wurde von ALFÖLDY im Rahmen einer Aufarbeitung des gesamten im 
Matronen-Heiligtum auf dem „Addig“ entdeckten Inschriftenmaterials wie folgt wiedergegeben: ${ }^{49}$

[V]acallinehis

[. N] umarian-

[iu]s Candidus

(pro se) et suis v(otum) l(ibens) m(erito)

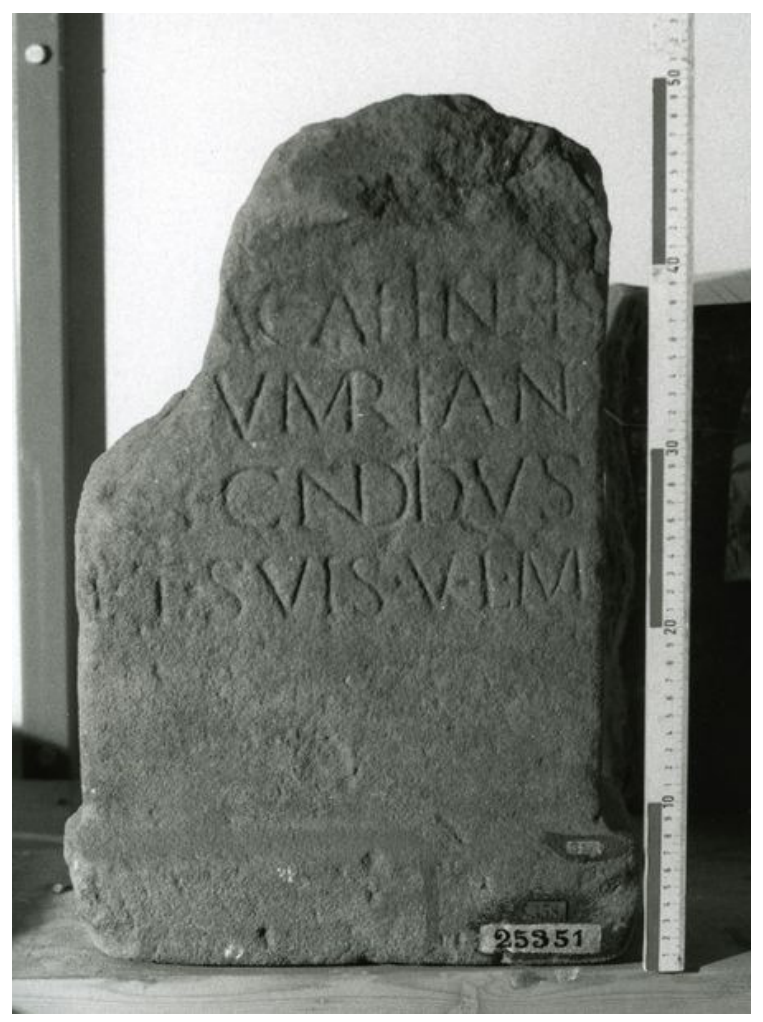

Abb.5: Fragmentarische Weihinschrift aus Nettersheim-Pesch: CIL XIII 12034

(C) CIL XIII/2-Projekt, Universitäten Trier/Flensburg)

Laut ALFÖLDY handelt es sich bei dem nicht weiter bezeugten Gentiliz Numarianius „Wohl“ um eine Verschreibung oder Variante des Namens Numerianius. ${ }^{50}$ Allerdings sollte der Name aufgrund der Platzverhältnisse am Beginn von Zeile 3, wenn überhaupt - wie schon von LEHNER vorgeschlagen ${ }^{51}-$, eher zu [N] umarian [u]s ergänzt werden. ${ }^{52}$ Es handelt sich bei dem Namen also höchstwahrscheinlich - ebenso wie auf der zuvor besprochenen Inschrift aus Eschweiler-Fronhoven - um ein Pseudogentiliz mit einer Endung auf-ianus.

Als Basis für den erhaltenen Namensrest VMARIAN[V]S kommen kelt. Namen wie Iantumarus, Iumarus, Litumarus, Lutumarus, Ritumarus, Vatumarus etc. in Frage. Aus dem Bestand der bekannten Namen aus den germanischen Provinzen

\footnotetext{
${ }^{49}$ ALFÖLDY 1968, 51. Nr.131. - Der Stein befindet sich heute im Rheinischen Landesmuseum in Bonn (Inv.-Nr.25351).

50 ALFÖLDY 1968, 51. Nr.131, 71. - [N] umarian[iu]s findet sich auch bei SoLIN/SALOMIES ${ }^{2}$ 1994, 128 und KaKoschKe 2006, 289-290. CN 872. Die EDH Nr.HD077145 bietet (wohl versehentlich) die Lesung Numarian[iu]s.

${ }^{51}$ LEHNER 1918, 162. Nr.358.

${ }^{52}$ S. auch CIL XIII 12034, EDCS Nr.12800055.
} 
bieten sich als Basis nur die Namen Lutumarus und Iumarus an. ${ }^{53}$ Dabei ist eine Ergänzung zu [Lut]umarian[u]s aufgrund der Platzverhältnisse am Beginn von Zeile 2 offenbar kaum möglich. Vergleichbares gilt für fast alle weiteren genannten Namen, sofern der Steinmetz nicht schon am Beginn der Zeile mit Ligaturen bzw. Nexus gearbeitet hat.

Hier wurde letztendlich eine Ergänzung zu [I] umarian[u]s mit einem vorangehenden Pränomen favorisiert. Die Ergänzung ist allerdings lediglich nicht unwahrscheinlich, aber keinesfalls sicher. Für ein Pränomen spricht jedoch zumindest die Tatsache, dass fast alle männlichen Dedikanten aus Nettersheim-Pesch mit einem Gentiliz auch ein Pränomen führen. ${ }^{54}$

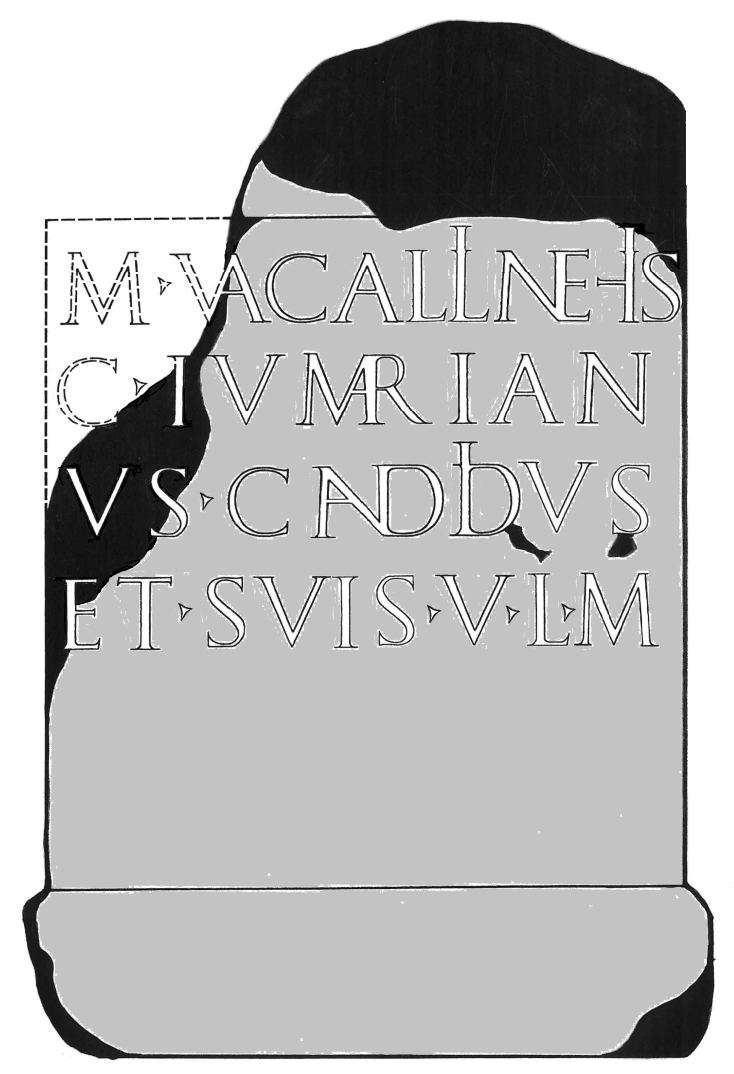

\begin{abstract}
Abb.6: Leicht idealisierte Rekonstruktionszeichnung der Weihinschrift CIL XIII 12034 aus Nettersheim-Pesch mit teilweise beispielhaften Ergänzungen (Zeichnung A.K.)
\end{abstract}

Wie eine Rekonstruktionszeichnung der Inschrift zeigt (Abb.6), kann auch die Lesung der ersten Zeile leicht korrigiert werden. Hier stand wohl zu Beginn noch ein M (oder eine MA-Ligatur) für Matronis und der Matronenbeiname wurde höchstwahrscheinlich - wie es vielfach auf den Weihungen aus Pesch zu sehen ist - mit einer VA-Ligatur eingeschlagen. ${ }^{55}$ Somit darf an dieser Stelle folgende Neulesung der Inschrift vorgeschlagen werden:

\footnotetext{
${ }^{53}$ Zu Lutumarus s. KAKOSCHKe 2007, 483. CN 1818. Zu Iumarus s. KAKOsChKE 2007, 437. CN 1639 (jeweils mit Belegen).

${ }^{54} \mathrm{Vgl}$. ALFÖLDY 1968, 80.

${ }^{55}$ Einen vergleichbaren Textanfang mit einem M (für Matronis) gefolgt vom Matronenbeinamen mit einer VA-Ligatur am Wortanfang bieten folgende Weihinschriften aus Nettersheim-Pesch: CIL XIII
} 


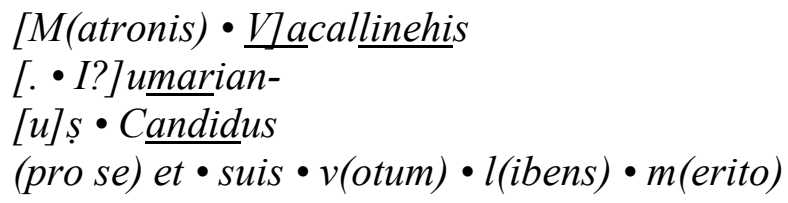

V.

Durch eine Zeichnung BROELMANNs aus dem frühen 17. Jahrhundert ist aus Köln/CCAA eine fragmentarische Grabinschrift bekannt (Abb.7), die nicht in das CIL aufgenommen wurde. B. und H. GALSTERER geben den Text des Steins, der sich nicht näher datieren lässt, aufgrund der alten Zeichnung wie folgt wieder: ${ }^{56}$

$$
\begin{aligned}
& \text { [---] Marian(i)us Ambactus [---] } \\
& \text { [ann(orum) ---] • stip(endiorum) XXII [---] }
\end{aligned}
$$

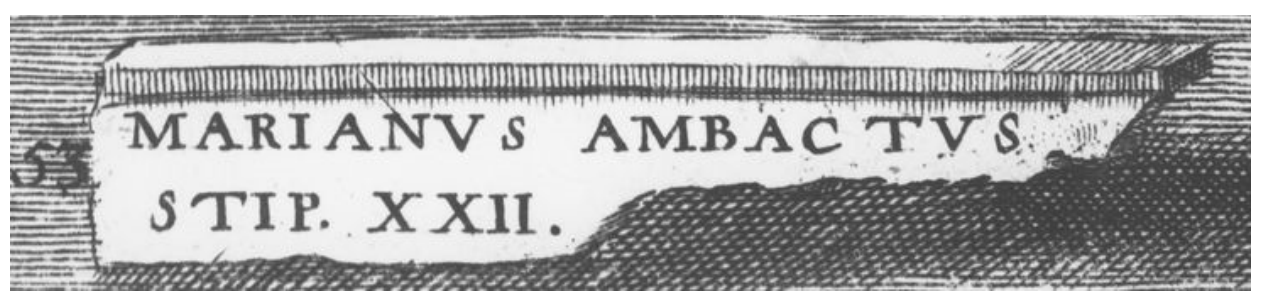

Abb.7: Fragmentarische Grabinschrift aus Köln/CCAA: IKöln ${ }^{2} \mathrm{Nr} .352$ (aus: BROELMANN 1608, Tab.III. Nr.53)

Im Kommentar zur Inschrift rechtfertigen B. und H. GALSTERER die Lesung des Gentilnomens mit den Worten: „Marianus könnte vielleicht eine ,Verlesung“ (NILigatur) für den seltenen Namen Marianius sein (CIL XIII 4402).“ Allerdings findet sich auf der Grabinschrift CIL XIII 4402 aus Metz/Divodurum nicht das Gentiliz Marianius, sondern nur der einfache Name Marianus. Auch Solin/SALOMIES führen keinen Beleg für ein Gentiliz Marianius. Obwohl ein solcher Name dennoch existiert haben mag - zumindest ist ein Pseudogentiliz Marianius, abgeleitet vom Cognomen Marianus theoretisch denkbar - sollte man vielleicht eher der Zeichnung BROELMANNs vertrauen. In diesem Sinne listen auch Solin/SALOMIES den Beleg aus Köln als Gentiliz Marianus auf. ${ }^{57}$ Für das Gentilnomen finden sich nur wenige weitere Belege. Offenbar handelt es sich bei dem Namen um ein seltenes italisches Gentiliz. ${ }^{58}$

Der äußeren Form nach könnte mit dem Beleg für Marianus aus Köln/CCAA aber ebenso ein einheimisches Pseudogentiliz vorliegen, ein peregriner-ianus-Name mit der Basis Marius oder Marus. ${ }^{59}$ Zumindest spricht das Cognomen Ambactus, das eindeutig keltischen Ursprungs ist, ${ }^{60}$ nicht gegen diese Auffassung.

12027 (= ALFÖLDY 1968, 35-36. Nr.11), 12031 (= ALFÖLDY 1968, 36-37. Nr.16), 1. N. 89. Nr.266 (= ALFÖLDY 1968, 38. Nr.25), ALFÖLDY 1968, 40. Nr.35 (= LEHNER 1918, 174. Nr.406).

${ }^{56}$ IKöln $^{2}$ 292. Nr.352 (mit Zeichnung) [= GALSTERER 1975, 70. Nr.287. Taf.63 (Zeichnung) = EDCS Nr.00500083 = EDH Nr.HD075850 (jeweils mit entsprechender Lesung)].

${ }^{57}$ SOLIN/SALOMIES ${ }^{2}$ 1994, 113. S. auch J. KLINKENBERG, BJb 108/109 (1902), 163. Nr.163, OPEL III 57.

${ }^{58}$ KAKOSCHKE 2006, 259. GN 744 (mit allen Belegen). Zum Namen s. auch SCHULZE 1904, 17.

${ }^{59}$ Zu den gängigen Namen s. KAKOSCHKE 2008, 84. CN 1921, 90-91. CN 1941.

${ }^{60}$ Zum Namen s. KAJANTO 1965, 313 (,gallic loan-word“), KAKOSCHKE 2007, 91. CN 153

http://www.fera-journal.eu 
Abschließend sei noch vermerkt, dass die eventuell etwas ungenaue Zeichnung BROELMANNs möglicherweise auch die Lesung des unvollständigen Namens [---] marianus Ambactus zulässt.

\section{VI.}

Im Jahre 1865 entdeckte man unweit des Herrenhauses Gripswald bei OssumBösinghoven (Stadt Meerbusch) einen fragmentarischen Weihestein, der heute verloren ist (Abb.8). Der Stein ist nur durch STOLLWERCK bekannt, der die Inschrift der Weihung in einer Publikation aus dem Jahre 1877 wie folgt wiedergab: ${ }^{61}$

\section{Matronis / Octocan/abus Q. Va/rianus ...}

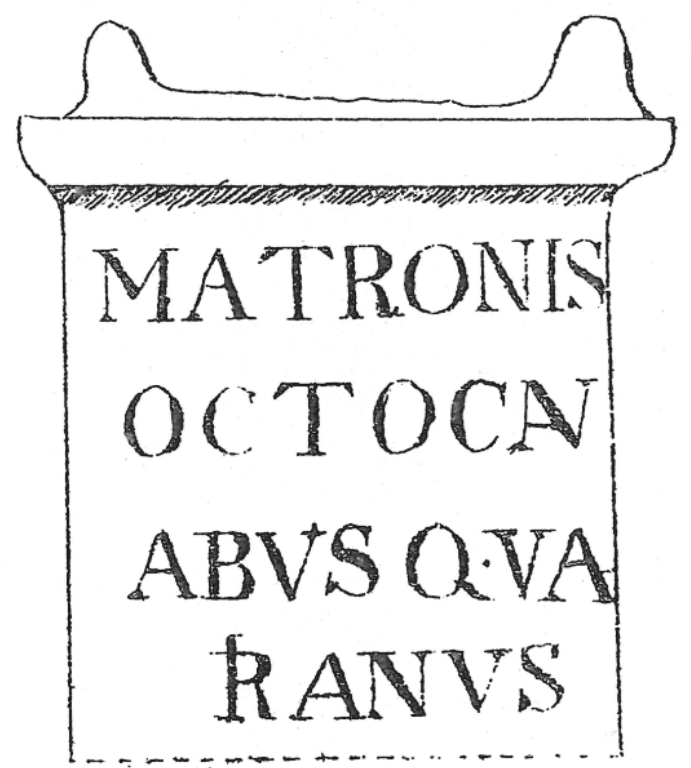

Abb.8: Fragmentarische Weihinschrift aus Ossum-Bösinghoven: CIL XIII 8574 (aus: STOLLWERCK 1877, Taf.1)

STOLLWERCK erblickte in dem von ihm entzifferten Varianus ein Gentiliz. Schon IHM bezweifelte die Lesung STOLLWERCKs. „Nach der Zeichnung ... zu urtheilen“, stellte IHM unter Bezug auf die Freistellung am Beginn von Zeile 4 fest, „,önnte der Name des Dedikanten auch Va[le]rianus gelautet haben. “62 Im Index des CIL wurde das Gentiliz Varianus zwar aufgenommen, jedoch als Hinweis auf eine möglicherweise fehlerhafte Überlieferung mit einem Stern gekennzeichnet. ${ }^{63}$ Dagegen nahm das OPEL den Namen zwar auf, allerdings als Cognomen. ${ }^{64}$ Für die Lesung des Namens Q(uintinius) Varianus (oder ähnlich) könnte immerhin die Tatsache sprechen, dass das vom Gentiliz Varius abgeleitete Varianus mehrmals als Cognomen bezeugt ist. ${ }^{65}$

\footnotetext{
${ }^{61}$ STOLLWERCK 1877, 173.

${ }^{62}$ M. IHM, BJb 83 (1887), 154. Nr.327. Zweifel an der korrekten Lesung STOLLWERCKs hegte gleichzeitig auch M. SIEBOURG, WZ 6 (1887), 281, der jedoch keine alternative Lesung vorschlug.

${ }^{63}$ CIL XIII/5 21 (Index). S. auch KAKOSCHKE 2006, 416. GN 1352 [„,Varianus (GN?)“]

${ }^{64}$ OPEL IV 147.

${ }^{65}$ S. auch KAKOSCHKE 2006, 416. GN 1352. - Zum Cognomen Varianus s. KAJANTO 1965, 158, OPEL IV 147 (mit dem Beleg aus Ossum-Bösinghoven).
} 
Ausgehend von der Tatsache, dass für das Gentiliz Varianus nur zwei weitere Belege aus Italien vorliegen, ${ }^{66}$ wurde kürzlich eine neue Lesung des überlieferten Textes vorgeschlagen. Demnach soll auf dem Stein der Name Q(uintus) Val(erius) [... rianus gestanden haben ${ }^{67}$ Folglich wird STOLLWERCK damit wiederum unterstellt, bei seiner Lesung Buchstaben übersehen zu haben, nämlich eine ligiertes L am Ende von Zeile 3 und einige Buchstaben am Anfang von Zeile 4.

Die Lesung STOLLWERCKs muss jedoch (zumindest angesichts des gelesenen Namens) nicht zwingend angezweifelt werden. Immerhin spricht STOLLWERCK selbst von ,gut gemeißelten Buchstaben“. ${ }^{68}$ Allerdings ist es eher unwahrscheinlich, dass der Dedikant aus Ossum-Bösinghoven, in dem man höchstwahrscheinlich einen Einheimischen erblicken kann, ein sehr seltenes italisches Gentiliz trug. Daher führte der Dedikant der Matronenweihung vielleicht ebenfalls ein einheimisches Pseudogentiliz, einen Namen auf-ianus. Die Basis für dieses erstmals bezeugte Pseudogentiliz wäre in diesem Fall das gängige Cognomen Varus. ${ }^{69}$

STOLLWERCK ging im unteren Bereich des Schriftfeldes von einer oder zwei fehlenden Zeilen aus. ${ }^{70}$ In diesen Zeilen werden dann wohl noch ein Cognomen und eine gängige Schlussformel gestanden haben.

\section{VII.}

Im Tal des Rotbaches zwischen Mühlheim-Wichterich und Erftstadt-Niederberg fand sich vor einigen Jahren ein schlanker Inschriftenstein (Höhe 70,2 cm - Breite 25,5 cm - Tiefe 22,4 cm) aus Drachenfelstrachyt, der in die Zeit zwischen 150 und 260 n. Chr, datiert werden kann (Abb.9). BAUCHHENß und TUTLIES legten als Erste eine Lesung der Inschrift vor, welche sich in doppelter (fast identischer) Ausführung auf der Vorder- (A) und der Rückseite (B) des Sockels befindet. Diese Lesung konnte im nachhinein hinsichtlich des eingeschlagenen Gentilnomens leicht korrigiert werden. Demnach lauten die Inschriften wie folgt: ${ }^{71}$

A1

$$
\begin{aligned}
& \text { EIVS[ca. } 3-4] C V R \\
& \text { C(aius) Gasp(e)na- }
\end{aligned}
$$$$
\text { sianus }
$$$$
\text { [C] assius }
$$

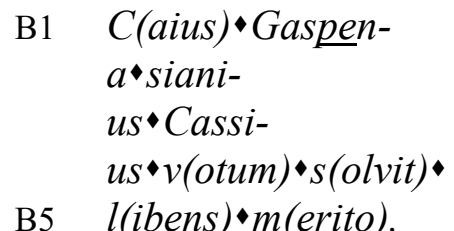

B5 l(ibens) m(erito).

BAUCHHENß und TUTLIES gehen davon aus, dass der Dedikant zunächst die Inschrift A in Auftrag gab. Jedoch schlug der Steinmetz die Inschrift zum Leidwesen des Auftraggebers fehlerhaft ein. Offenbar hat dann ein zweiter Steinmetz (mit weniger akkuraten Buchstaben, einer anderen Laufweite der Buchstaben und schief gesetzten Zeilen) auf der Rückseite des Steins eine korrigierte Fassung des Textes mit Worttrennern angebracht (B). Lautete der Name des Dedikanten in der älteren Inschrift noch C(aius) Gasp(e)nasianus Cassius, so wurde er jetzt als C(aius) Gaspenasianius Cassius eingeschlagen. Der nicht besonders qualifizierte Steinmetz ergänzte also ein I

\footnotetext{
${ }^{66}$ CIL VI 28332 (Rom), XI 5981 [= I 2122 = VI 28389 (Cagli/Ad Calem)]. Zum Namen s. SCHUlZE $1904,377$.

${ }^{67}$ A. KAKOSCHKE, FeRA 32 (2017), 6-8. Nr.3. Abb.5 (Zeichnung).

${ }^{68}$ STOLLWERCK 1877, 173.

${ }^{69} \mathrm{Zu}$ Varus s. KAKOSCHKE 2008, 427-428. CN 3222.

${ }^{70}$ STOLLWERCK 1877, 173.

71 BauchHenß/Tutlies 2006, 134-137. Abb.131-133 (Photos) (= AE 2006, 869), A. KaKoschKe, FeRA 22 (2013) 1-4 (= AE 2013, 1114). - Der Stein lagert heute im Rheinischen Landesmuseum in Bonn (Inv.-Nr. E 2006 / 75).
} 
im Namensteil SIANVS und ein E im Namensteil GASPNA, das er (vielleicht erst nachträglich) mit dem P ligierte. ${ }^{72}$ Diese Interpretation der Ersteditoren wurde auch vom Autor dieser Zeilen geteilt, der in Gaspenasianius die korrekte Form des Gentilnomens erblickte. ${ }^{73}$
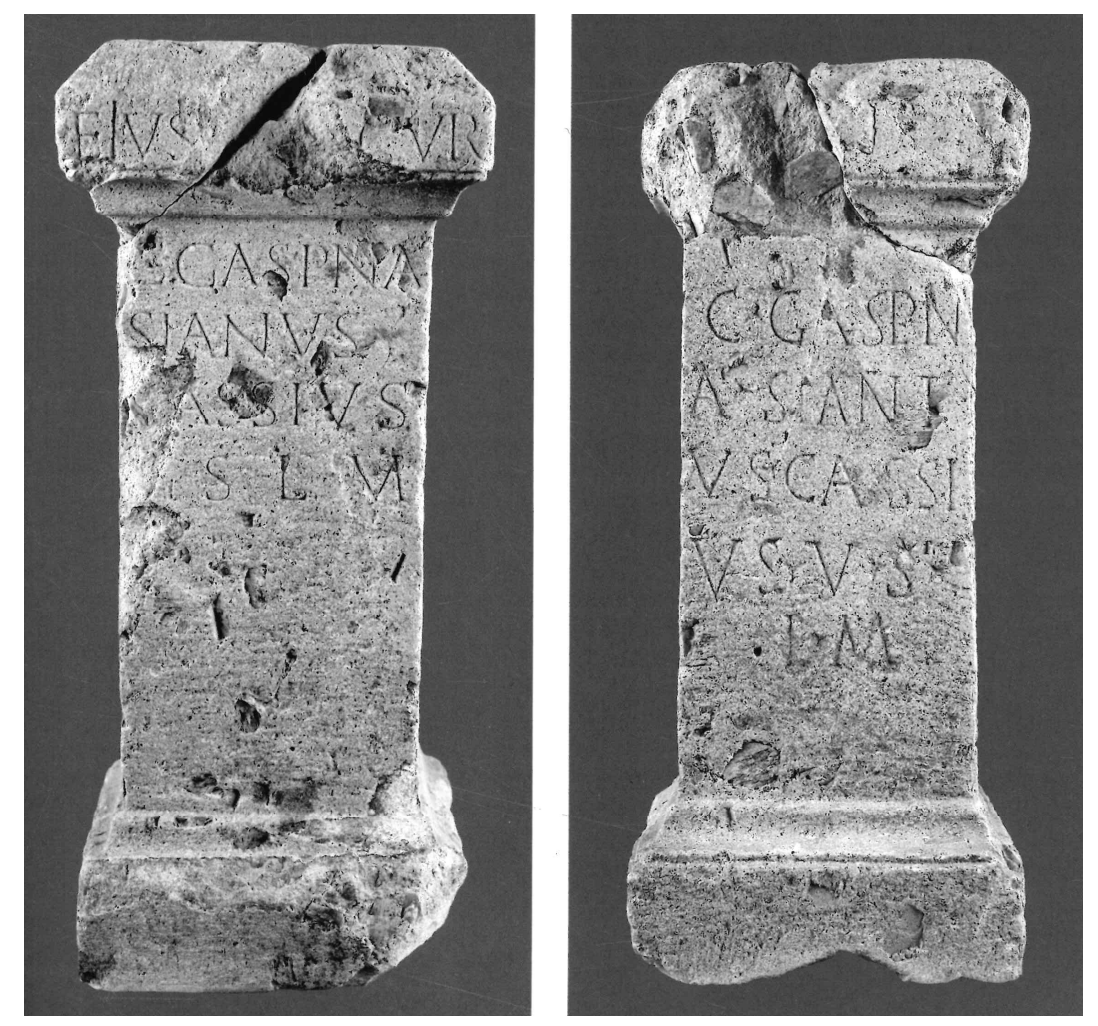

Abb.9: Weihinschrift aus Erftstadt-Niederberg: AE 2013, 1114 (aus: Archäologie im Rheinland 2006, 135. Abb.131-132)

$\mathrm{Zu}$ Recht geben BAUCHHENß und TUTLIES angesichts der Ausführungen der Inschriften jedoch zu bedenken, dass der Steinmetz der korrigierenden Fassung B „deutlich schlampiger“ gearbeitet hat als der Steinmetz der Fassung A. BAUCHHENß und TUTLIES vermerken zu Recht, dass die Fehler des ersten Handwerkers noch nachträglich „mit einem dünnen Stucküberzug und richtiger Schreibung mit Farbe“ hätten behoben werden können. ${ }^{74}$ Allerdings wäre eine Korrektur mit Hilfe von Ligaturen viel einfacher (und gängiger) gewesen. So hätte der erste Steinmetz (wie sein Nachfolger) das vergessene $\mathrm{E}$ mit dem $\mathrm{P}$ verbinden können. Und durch eine Verlängerung der rechten Längshaste des $\mathrm{N}$ in SIANVS wäre er relativ leicht zu SIANIVS gelangt. ${ }^{75}$

Da es sich entgegen älteren Überzeugungen auch bei Gaspenasianus, einem typischen Pseudogentiliz auf -ianus, um den korrekten Namen des Dedikanten gehandelt haben kann, darf man sicherlich auch in der sorgfältiger gestalteten Seite A eine korrigierende Fassung des Textes B erblicken. Laut BAUCHHENß und TUTLIES bleibt es zudem nicht ausgeschlossen, dass die Basis, wie auch andere Steine, mit Absicht auf beiden Seiten beschriftet wurde, da sie für eine „doppelansichtige Auf-

\footnotetext{
${ }^{72}$ BAUCHHENß/TUTLIES 2006, 135-136.

${ }^{73}$ A. KAKOSCHKE, FeRA 22 (2013), 2.

${ }^{74}$ BAUCHHENß/TUTLIES 2006, 136.

${ }^{75}$ A. KakoschKe, FeRA 22 (2013), 2. Anm.2.
} 
stellung“ vorgesehen war. ${ }^{76}$ In diesem Fall müsste man sich natürlich fragen, ob mit einer der Inschriften überhaupt eine korrigierende Fassung vorliegt. Diese nicht unwahrscheinliche Vermutung mag zu der Frage führen, ob die Formen Gaspenasianus und Gaspenasianius nicht als mehr oder weniger gleichwertig angesehen wurden. Immerhin liegen mit beiden Formen Namen vor, die auf den germanischen Namen *Gaspenasus zurückzuführen sind. Und die semantische Füllung beider Namen ist identisch. Beide Pseudogentilnomina stehen als Patronymika für „Sohn des Gaspenasus". Insofern lassen sich vielleicht auch die in Niedergermanien mehrfach bezeugten (konkurrierenden) Formen Bassianus und Bassianius, ${ }^{77}$ die entsprechend beide mit „Sohn des Bassus“ zu übersetzen sind, trotz unterschiedlicher Bildungsweisen als nahezu identische Namen auffassen. ${ }^{78}$ Die aufgeworfenen Fragen können jedoch nur mit Hilfe weiterer epigraphischer Neufunde geklärt werden.

\section{VIII.}

Aus Öhringen/vicus Aurelianus (Hohenlohekreis) stammt ein fragmentarischer Altar (Höhe $40 \mathrm{~cm}$ - Breite $44 \mathrm{~cm}$ - Tiefe $19 \mathrm{~cm}$ ) aus Sandstein, der im Jahre 1861 gefunden wurde (Abb.10). In der EDH wird der eingeschlagene Text des Steins, der aufgrund der angegebenen Datierung am 1. September des Jahres 169 n. Chr. geweiht wurde, wie folgt angegeben: ${ }^{79}$

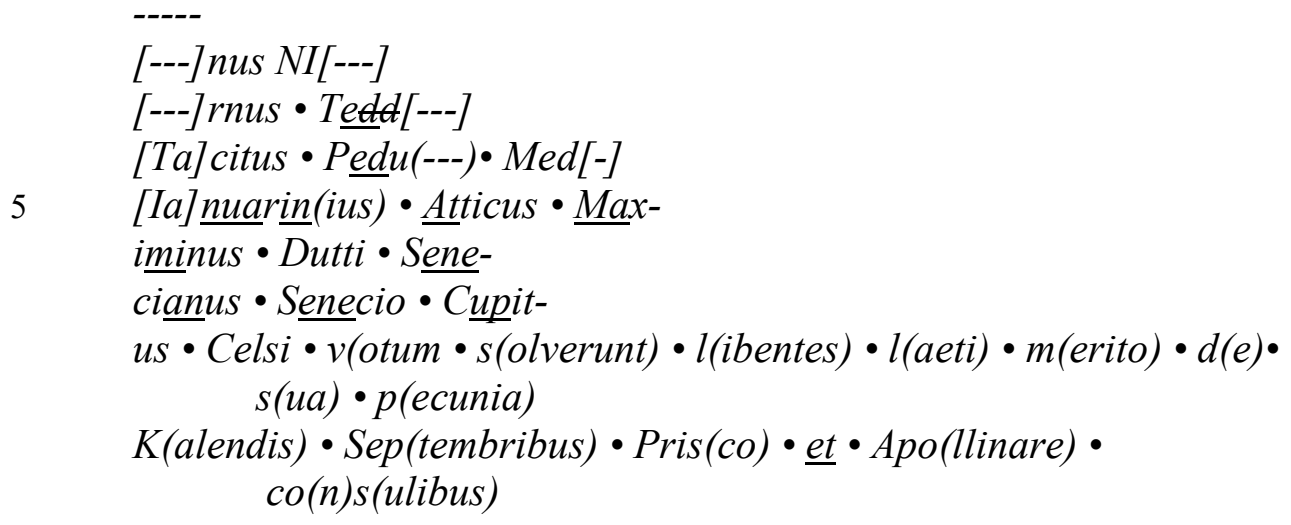

\footnotetext{
${ }^{76}$ BAUChHEN/TUTLIES 2006, 136. - Verwiesen sei hier auf folgende Steine mit einer doppelten Beschriftung: CIL XIII 6709 (= KAKOSCHKE 2002, 281-282. Nr.2.62) (Mainz/Mogontiacum), 8182 [= IKöln ${ }^{2}$ 56-57. Nr.34 (mit Photos)], 8371 [= IKöln² 381-382. Nr.461 (mit Photos)] (beide Köln/CCAA), $11615 \mathrm{c} \quad[=\mathrm{EDH}$ Nr.HD075161 (Strasbourg-Koenigshoffen/Argentorate)], 11809 (= EDH Nr.HD075205) (Mainz/Mogontiacum), 11938 (= EDH Nr.HD075836) (Niedernberg), AE 1984, 654 [= IKöln² 102-103. Nr.94 (mit Photos)] (Köln/CCAA), 1996, 1152 (= EDH Nr.HD036702) (Osterburken). ${ }^{77} \mathrm{Zu}$ den Namen s. KAKOSCHKE 2006, 106. GN 197 (mit sämtlichen Belegen).

${ }^{78}$ Die leicht unterschiedliche Schreibung gleicher Namen ist durch weitere Inschriften bezeugt, sofern die genannten Personen tatsächlich identisch sind. Verweisen sei auf CIL XIII 12019 (= ALFÖLDY 1968, 43. Nr.63) aus Nettersheim-Pesch (Laubasn[i]us Exomni [fil.]) und 1. N. 89. Nr.266 (= ALFÖLDY 1968, 38. Nr.25) aus Nettersheim-Pesch [Leubasnius Exomni (f.)]. - S. ferner die Formen [Neg]aletianus und Negalatianus in der hier zuvor besprochenen Inschrift RÜGER 1983, 154-156. Nr.39 aus Eschweiler-Fronhoven.

${ }^{79}$ EDH Nr.HD054427. Worttrenner und Ligaturen wurden vom Autor ergänzt. - Der Stein befindet sich heute im Limesmuseum in Aalen (Inv.-Nr.RL 190) (Leihgabe des Württembergischen Landesmuseums Stuttgart).
} 


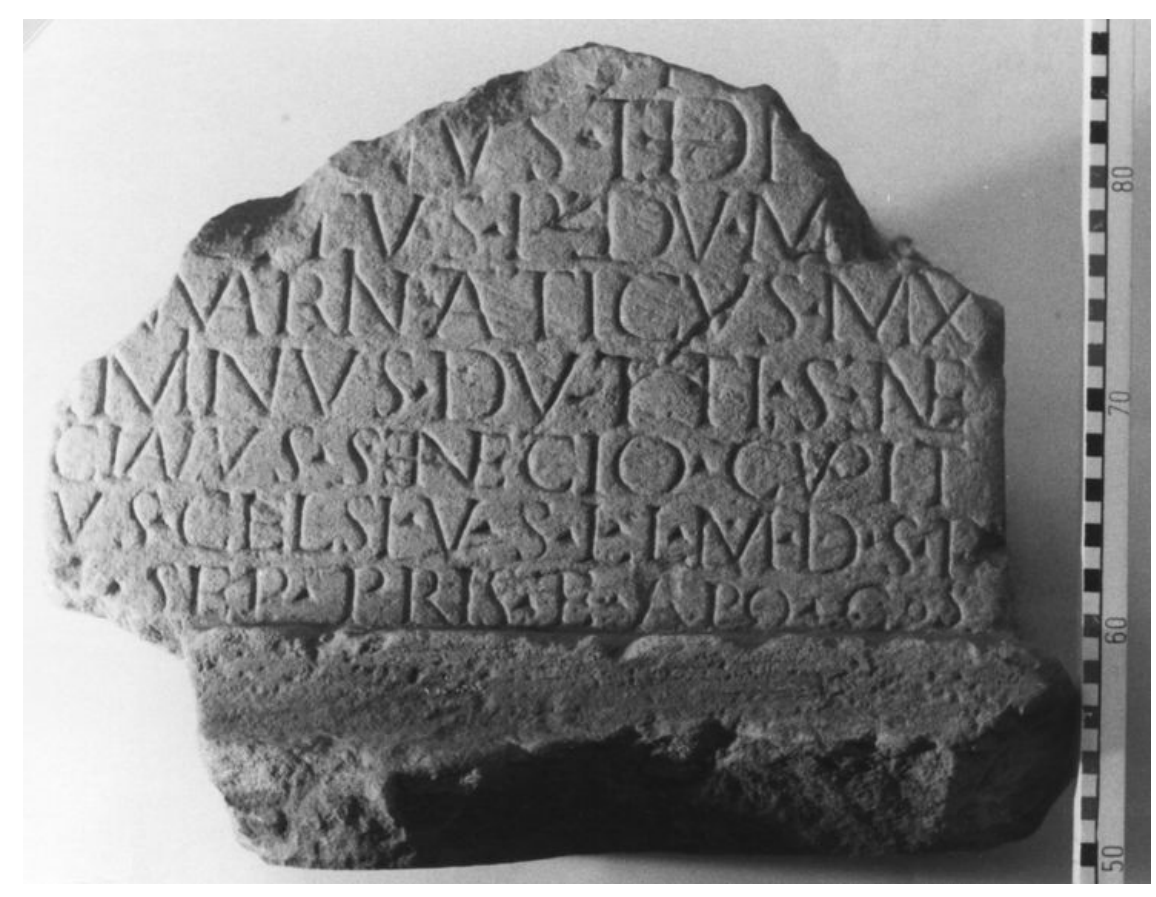

Abb.10: Fragmentarische Weihinschrift aus Öhringen: CIL XIII 6544 (C) CIL XIII/2-Projekt, Universitäten Trier/Flensburg)

Die Inschrift wurde von mehreren Dedikanten einer unbekannten Gottheit geweiht. In den Weihenden darf man möglicherweise die Mitglieder eines Vereins erblicken. Die Lesung der fragmentarischen und teilweise abgekürzten Namen der ersten Zeilen bleibt größtenteils ungewiss. Sicheren Boden betritt man ab der vierten erhaltenen Zeile mit den Namen Ianuarinius Atticus und Maximinus Dutti (filius). Der folgende Name wird im CIL mit Senecianus Senecio(ni? filius) (sic) wiedergegeben. ${ }^{80}$ Diese Lesung favorisieren grundsätzlich weitere Bearbeiter der Inschrift. ${ }^{81}$ Dagegen erblickten die Mitarbeiter der EDH (nach Ausweis der Angaben im kommentierenden Datenbank-Feld „Personen“) in Senecianus Senecio offenbar zwei Personen und in dem anschließend genannten Cupitus Celsi eine weitere Person.

Beide Auffassungen sind wohl nicht korrekt. M.E. bieten sich zwei überzeugendere Lesungen an. Entweder lauten die Namen Senecianus (et) Senecio (et) Cupitus Celsi (filii), oder es sind die Namen Senecianus Senecio und Cupitus Celsi (filius) zu lesen. In letzterem Fall läge mit Senecianus offenbar ein weiterer Beleg für ein peregrines Pseudogentiliz auf -ianus vor. Die Basis für das Pseudogentiliz war dann gegebenenfalls der (Vater-)Name Senex. Die Lesung/Interpretation des Namens Senecianus bleibt jedoch unsicher.

IX.

Vergleichbares gilt für einen Namen auf einem Weihestein aus Wissembourg (dép. Bas-Rhin). Die Inschrift wurde im Jahre 1733 in Zweitverwendung im Château SaintRémy entdeckt. Nach seiner Auffindung gelangte der Altar nach Strasbourg und wurde dort während der deutschen Belagerung der Stadt im Jahre 1870 zerstört. Der

\footnotetext{
${ }^{80}$ CIL XIII 6544.

${ }^{81}$ HAUG/SIXT ${ }^{2}$ 1914, 618-619. Nr.434 (mit Photo), RIESE 1914, 363. Nr.3610, KEMKES/WILlbURGER 2004, 39. Abb.34 (Photo), 108. Nr.34, KAKoschKe 2008, 315. CN 2783-7, CN 2784-14, LUPA Nr.7666 (mit Photo).
} 
Text des Steins ist jedoch u.a. durch eine Abbildung ScHÖPfLINs bekannt (Abb.11). Gemäß den Angaben im CIL lautete die Inschrift wie folgt: ${ }^{82}$
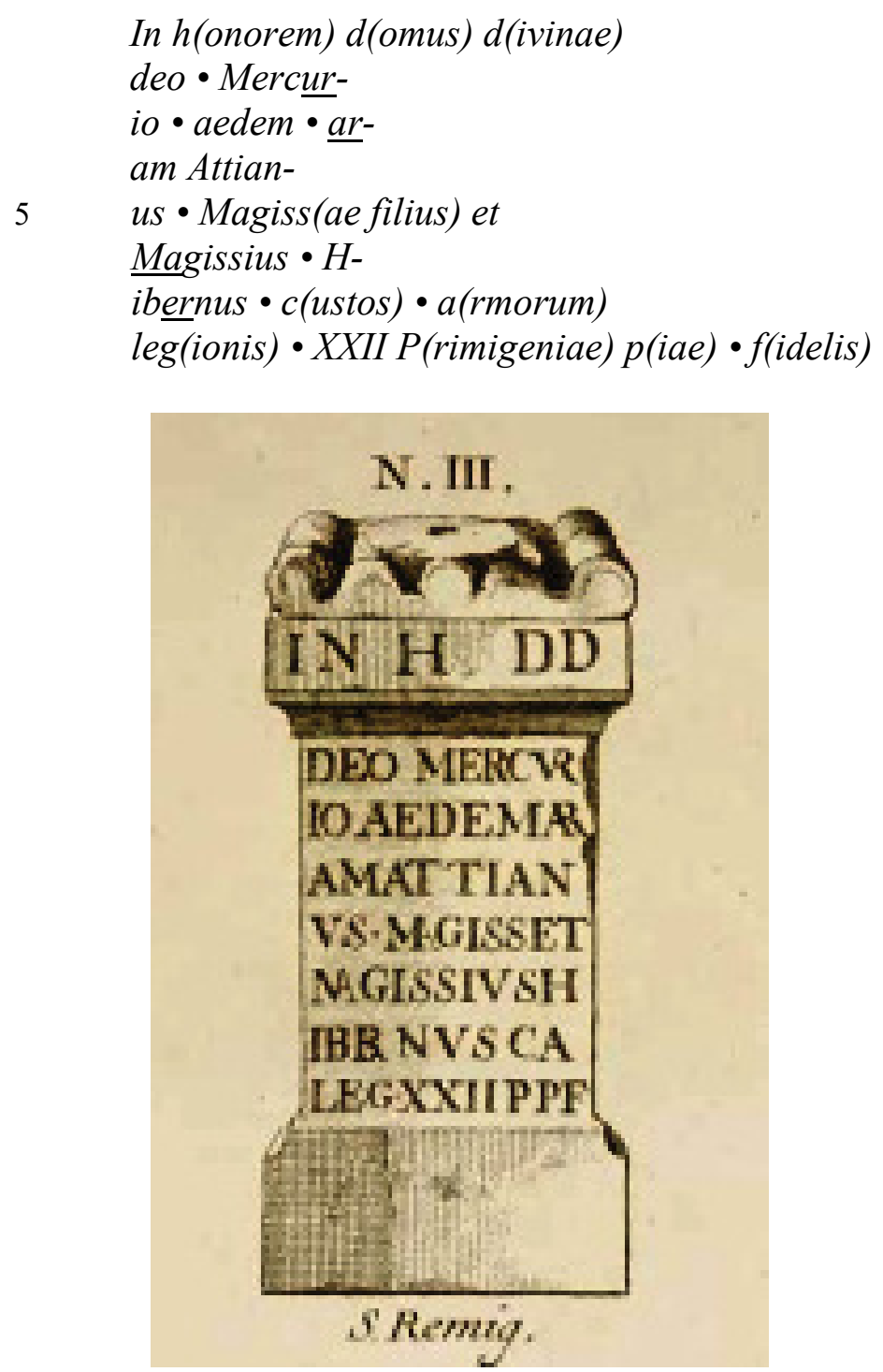

Abb.11: Weihinschrift aus Wissembourg: CIL XIII 6078 (aus: SCHÖPFLIN 1751, 437. Taf.1/3)

Die Lesung der Inschrift bereitet nur an einer Stelle Schwierigkeiten. In Zeile 5 sollte MAGISS gemäß den kommentierenden Angaben im CIL zu Magissae filius aufgelöst werden. Dieser Lesung haben sich - offenbar im Bewusstsein, dass ein italisches oder einheimisches Gentiliz Attianus nicht existiert - fast alle folgenden Bearbeiter der Inschrift angeschlossen. ${ }^{83}$ Demnach wurde der Stein offensichtlich von zwei Brüdern dediziert, von Attianus, dem Sohn des Magissa, der anscheinend kein römisches Bürgerrecht besaß, und von Magissius Hibernus, der als römischer Bürger in der legio XXII Primigenia in Mainz/Mogontiacum diente.

Bei Attianus kann es sich aber sehr wohl um ein Gentiliz handeln, nämlich um ein Pseudogentiliz auf -ianus, das sicherlich vom Cognomen bzw. Nomen simplex

\footnotetext{
${ }^{82}$ CIL XIII 6078 [= BRAMBACH 1867, 333. Nr.1836 (jeweils mit weiteren Literaturhinweisen)].

${ }^{83}$ S. u.a. SPICKERMANN 2003, 345. Nr.23, RAEPSAET-CHARLIER 2006, 364, KAKOSCHKE 2007, 132. CN 356-8, KAKOSCHKE 2008, 63. CN 1846-1, EDH Nr.HD079176, EDCS Nr.11000070.
} 
Attius abgeleitet wurde. Ob RIESE, der die Lesung des Namens Attianus Magiss(a) favorisierte, bereits an eine solche Bildung dachte, sei dahingestellt. ${ }^{84}$ Auf jeden Fall führt auch das OPEL den vorliegenden Beleg für Attianus als (nur einmal bezeugtes) Gentilnomen. ${ }^{85}$ Dieser immer noch unsicheren Auffassung zufolge darf man in dem in der Inschrift zuerst genannten Attianus Magissa den Vater des Magissius Hibernus erblicken, der seiner Nomenklatur zufolge wohl ebenfalls schon über das römische Bürgerrecht verfügte.

$\mathbf{X}$.

Abschließend sei auf einen fragmentarischen Weihestein aufmerksam gemacht, der sich im Jahre 1826 bei der Heilquelle Heilstein bei Wollseifen (Nationalpark Eifel) fand. Die heute verlorene Inschrift lässt sich infolge fehlender Anhaltspunke zeitlich nicht näher einordnen. Aufgrund der 1826 publizierten Zeichnung von Hons (Abb.12) nahm DoMASZEWSKI den Text des Steins (mit kleinen Abweichungen) im CIL wie folgt auf: ${ }^{86}$

\section{..LAILON \\ MADDGARISI- \\ ANVS BATAVS}

Offenbar handelt es sich bei dem Fragment um den Mittelteil eines Weihesteins. Unterhalb der letzten Zeile waren anscheinend keine Buchstaben mehr zu erkennen. Möglicherweise stand hier noch eine Schlussformel wie L(ibens) M(erito) mit weit auseinander stehenden Buchstaben. Die ersten Zeilen des Steins, in denen wohl der Name der Gottheit genannt wurde, können nicht mehr ergänzt bzw. sinnvoll gelesen werden. ${ }^{87}$ Den Namen des Dedikanten geben die einzelnen Bearbeiter der Inschrift unterschiedlich wieder. So lesen RIESE und BYVANCK im Gegensatz zum CIL den Namen Madd(---) Garisianus. $^{88}$ Für Maddgarisianus votiert u.a. DelamarRE. ${ }^{89}$ Dagegen schlägt DERKs die Lesung des Namens M. Add(ius) Garisianus vor. ${ }^{90}$ Einig sind sich alle Bearbeiter der Inschrift jedoch darin, dass es sich bei BATAVS um eine Herkunftsangabe handelt.

\footnotetext{
${ }^{84}$ RIESE 1914, 135. Nr.1208. Allerdings löst RIESE in Unkenntnis der korrekten Form des keltischen Cognomens zu Attianus Magiss(us) auf. Vgl. auch KAKOschKe 2006, 91. GN 152-1 [Attian(i?)us Magiss(us)].

${ }^{85}$ OPEL I $^{2}$ 89. Bei SOLIN/SALOMIES ${ }^{2}$ findet sich kein Beleg für ein Gentiliz Attianus.

${ }^{86}$ CIL XIII 7833. - Wie ein Vergleich mit Hons' Zeichnung (Abb.12) zeigt, ist die Wiedergabe DOMASZEWSKIs nicht ganz korrekt. So vermerkt DOMASZEWSKI nicht das kleine eingeschriebene A im Buchstaben G (Zeile 2). In Zeile 3 ist vor BATAVS anscheinend noch ein kleiner Worttrenner zu erkennen. Ferner kann die Lesung LAILON in Zeile 1 nicht zutreffen. Aufgrund des angegebenen Abstandes der Buchstaben ist eher ---LAPLON oder ---EAPLON zu lesen. Auch diese Buchstabenreste lassen jedoch keine sinnvolle Lesung zu.

${ }^{87}$ S. auch WHATMOUGH 1970, 856, nach dem ,,]lailon“ einen germanischen Eindruck macht.

${ }^{88}$ RIESE 1914, 269. Nr.2419, BYVANCK 1935, 509. Nr.1344.

${ }^{89}$ Delamarre 2007, 122. S. auch Weisgerber 1968, 25. Nr.57, 149 („Mit dem ... Batavernamen Maddgarisianus ... ist nicht viel anzufangen.“), KAKOSCHKE 2002, 91-92. Nr.1.62, KAKOSCHKE 2008, 61. CN 1833.

${ }^{90}$ DERKS 2004, 68. Nr.48.
} 


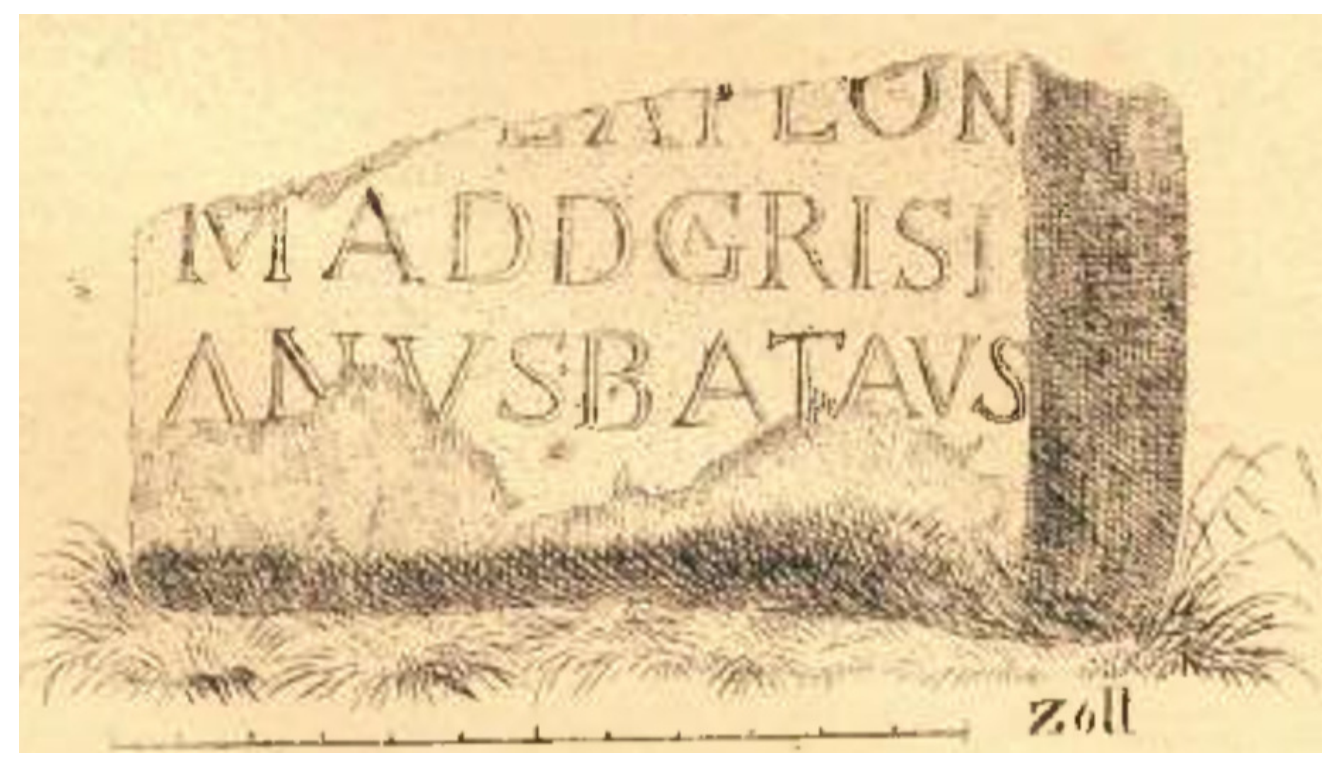

Abb.12: Fragmentarische Weihinschrift aus Heilstein bei Wollseifen: CIL XIII 7833 (aus: Hons 1826, 18. Taf.1. Abb.3)

Ausgehend von der Auffälligkeit, dass der Dedikant möglicherweise nur einen einfachen Namen führte, ohne peregrine Filiation oder Gentilnomen, gleichzeitig aber eine Herkunftsangabe gibt, lässt sich der überlieferte Name vielleicht auch als Maddgarisianus Bata(v)us [oder M. Addgarisianus Bata(v)us] lesen. Möglicherweise handelt es sich bei Maddgarisianus nicht um einen entstellt überlieferten Namen oder ein Nomen simplex, sondern wiederum um ein Pseudogentiliz auf-ianus. Der Name fügt sich auf jeden Fall gut in die Reihe einiger bisher bekannter-ianus-Namen ein, wie Laubasnianus, [I?]umarianus, Gaspenasianus, Ucsperianus oder Negalatianus, die alle eine einheimische Basis aufweisen, recht lang und etwas sperrig sind.

Das Cognomen des Dedikanten kann dafür sprechen, dass der Mann ein Bataver war oder batavische Vorfahren besaß. Wahrscheinlich wird er sich in Heilstein als Kurgast aufgehalten haben. Wo sein aktueller Wohnort lag, bleibt unbekannt. Auf jeden Fall muss es sich bei dem Dedikanten nicht zwangsläufig um eine aus dem Gebiet der Bataver angereiste Person handeln.

Mit dem Beleg aus Heilstein liegen insgesamt 31 Nachweise für Pseudogentilnomina auf -ianus vor (Tabelle 1). Einige Zeugnisse bleiben dabei wie gesehen fraglich. Solin/SALOMIES führen insgesamt 180 Gentilnomina auf -ianus auf. ${ }^{91}$ Bei den meisten dieser Namen handelt es sich um italische Gentilnomina, die entweder nur in Italien auftreten oder außerhalb Italiens durch Italiker bezeugt sind. So geht ein schon oben erwähnter Beleg aus dem frühen ersten Jahrhundert n. Chr. für das Gentiliz Marcianus aus Köln/CCAA auf den Erben eines italischen Legionärs zurück, der sicher ebenfalls aus Italien stammte. ${ }^{92}$

Am Rande sei bemerkt, dass einige der bei Solin/SALOMIES aufgeführten -ianus-Namen wie z.B. Aurelianus, Claudianus, Fuscianus, Sallustianus, Sergianus oder Vettianus nur im Osten des Imperiums und fast immer auf griechischen In-

\footnotetext{
${ }^{91}$ SOLIN/SALOMIES ${ }^{2}$ 1994, 276-277.

92 CIL XIII 8288 [= IKöln ${ }^{2}$ 273. Nr.322 (mit Photo)]. Laut B. und H. GALSTERER stammte der Mann angesichts seiner Tribus (Lemonia) vielleicht aus Bologna/Bononia.
} 
schriften in Erscheinung treten. Es wird sich bei diesen Namen also wohl kaum um italische Gentilnomina handeln. ${ }^{93}$

Eine Verteilungskarte für die einheimischen Pseudogentilnomina auf -ianus (Abb.13.) zeigt einen eindeutigen geographischen Schwerpunkt für die Namen. Die Mehrheit der Belege stammt aus dem Gebiet der $C C A A$. Dabei lassen sich in Köln, dem Hauptort der $C C A A$, nur wenige Belege nachweisen, die zudem alle unsicher bleiben. Die meisten Nachweise stammen also nicht aus dem multikulturellen Zentrum, sondern aus dem ubisch geprägten Hinterland der Kolonie. Insofern darf man in der Namensform mit Vorsicht eine ubische Besonderheit erblicken.

Außerhalb des ubischen Siedlungsgebietes treten die charakteristischen Pseudogentilnomina auf -ianus in Niedergermanien kaum auf. Ein Beleg stammt aus dem unmittelbar nördlich angrenzenden Gebiet der Kolonie Xanten/CUT, aus Meerbusch. Zwei Nachweise sind aus dem Handelsplatz Colijnsplaat/Ganuenta an der Nordsee bekannt. Angesichts der vielen aus Colijnsplaat/Ganuenta bekannten Agrippinenser, ${ }^{94}$ ist es allerdings nicht allzu gewagt, eine Herkunft der (verwandten?) Händler namens C. Sabinianus Saturninus und C. Sabinianus Sextus aus dem Gebiet $\operatorname{der} C C A A$ zu vermuten.

\begin{tabular}{|l|l|l|l|l|}
\hline Nr. & -ianus-Name & Basis & Fundort & Nachweis \\
\hline 1 & Appianus & Appius & (Blankenheim) & CIL XIII 7964 \\
\hline 2 & Appiannus! & Appius & Odendorf & CIL XIII 7978 \\
\hline 3 & Aprianus & Aper & Kerpen-Blatzheim & CIL XIII 8512 \\
\hline 4 & Apriana & Aper & Dormagen & CIL XIII 8525 \\
\hline 5 & Attianus & Attius & Wissembourg (F) & CIL XIII 6078 \\
\hline 6 & Atticianus(?) $)^{95}$ & Atticus & (Blankenheim) & CIL XIII 8845 \\
\hline 7 & Bassianus & Bassus & Nettersheim-Pesch & 4. N. Nr.150 \\
\hline 8 & Bassianu[s] & Bassus & Eschweiler-Fronhoven & AE 1984, 676 \\
\hline 9 & Bassiana & Bassus & Bonn & 2. N. Nr.172 \\
\hline 10 & Bassiana & Bassus & Titz-Rödingen & CIL XIII 7890 \\
\hline 11 & Bass[i]ana & Bassus & Titz-Rödingen & CIL XIII 7890 \\
\hline 12 & Bassiana & Bassus & Worms & 2. N. Nr.78 \\
\hline 13 & Gasp(e)nasianus & *Gaspenasus & Erftstadt & AE 2013 1114 \\
\hline 14 & Iabitiana & *Iabitus & Jülich & CIL XIII 7873 \\
\hline 15 & {$[$ I?]umarian[u]s } & Iumarus & Nettersheim-Pesch & CIL XIII 12034 \\
\hline 16 & Laubasnianus & Laubasnius & Morken-Harff & 3. N. Nr.233 \\
\hline 17 & Maddgarisianus & *Maddgarisus & Wollseifen & CIL XIII 7833 \\
\hline 18 & Marianus & Mar(i)us & Köln & IKöln ${ }^{2}$ Nr.352 \\
\hline 19 & Negalatianus(?) & *Negalatus & Eschweiler-Fronhoven & AE 1984, 693 \\
\hline 20 & {$[$ Neg]aletianus(?) } & *Negalatus & Eschweiler-Fronhoven & AE 1984, 693 \\
\hline 21 & Ovinciana96 & *Ovincus & Stockstadt am Main & AE 1992, 1286 \\
\hline
\end{tabular}

\footnotetext{
${ }^{93}$ Verwiesen sei in diesem Zusammenhang auf einen Centurio der in Bonn/Bonna stationierten legio I Minervia namens Claudianus Maximus aus Vitolišta in der Provinz Macedonia [AE 1979, 563 (= EDH Nr.HD026335)]. Auf den ersten Blick könnte man in dem Mann aufgrund seines Gentilnomens vielleicht einen Einheimischen aus Niedergermanien vermuten. Allerdings handelt es sich bei Claudianus nicht um ein einheimisches Pseudogentiliz auf -ianus. Nach Solin/SALOMIES ${ }^{2}$ 1994, 56 tritt der Name noch mehrmals (ausschließlich) im griechischen Osten des Reiches auf. Claudianus Maximus stammte daher höchstwahrscheinlich aus dem griechisch geprägten Raum des Imperiums.

${ }^{94}$ KAKOSCHKE 2002, 513-515, 591.

${ }^{95}$ Der Text der verlorenen Weihinschrift CIL XIII 8845 (= BRAMBACH 1867, 352. Nr.1980) aus der Sammlung Blankenheim ist nicht eindeutig überliefert. H. CROMBACH, Annales ecclesiastici et civiles metropolis Coloniae Agrippinensis, Hdschr. des Kölner Stadtarchivs, Chroniken und Darstellungen Nr.109, 65 liest den Dedikantennamen Attiganus [S]peratus. Dagegen bietet WILTHEIM 1842, Taf.8/20 (Zeichnung) die Lesung des Namens Atticianus [S]peratus. Zum nicht weiter bezeugten Attiganus s. KAKOSCHKE 2006, 91-92. GN 155. - Vielleicht darf man mit Vorsicht vermuten, dass auf dem Stein ATTICIANVS stand, mit einem CI-Nexus, einem kleinen I, das in das C eingeschrieben war.
}

http://www.fera-journal.eu 
Frankfurter elektronische Rundschau zur Altertumskunde 38 (2019)

\begin{tabular}{|c|c|c|c|c|}
\hline 22 & Sabinian(us?) & Sabinus & Strasbourg (F) & 1. N. Nr.137 \\
\hline 23 & Sabinianus ${ }^{97}$ & Sabinus & Iversheim & CIL XIII 7943 \\
\hline 24 & Sabinianus & Sabinus & Colijnsplaat (NL) & AE 1973, 371 \\
\hline 25 & Sabinianu[s] & Sabinus & Colijnsplaat (NL) & AE 1975,656 \\
\hline 26 & Senecianus & Senex & Öhringen & CIL XIII 6544 \\
\hline 27 & Simplicianus(?) ${ }^{98}$ & Simplicius & Köln & CIL XIII 8423 \\
\hline 28 & Tattianus & Tata oder Tatius & Inden-Pier & AE 2001, 1429 \\
\hline 29 & Titianus & Titus & Tivoli (I) $)^{99}$ & AE 2006, 394 \\
\hline 30 & Ucsperianus! & Uxperus & Jülich & CIL XIII 7877 \\
\hline 31 & Varianus & Varus & Meerbusch & CIL XIII 8574 \\
\hline
\end{tabular}

Tabelle 1: Belege für Pseudogentilnomina auf -ianus aus den zwei germanischen Provinzen

In der Provinz Germania superior lassen sich nur fünf Pseudogentilnomina auf -ianus ausmachen. Jedoch bleiben vier Belege unsicher und ein Beleg geht auf eine Frau namens Bassiana Tacita aus Worms/Borbetomagus zurück, die höchstwahrscheinlich aus dem Gebiet der CCAA stammte. ${ }^{100}$

Darüber hinaus sind im Großraum Gallien keine sicheren Belege für Pseudogentilnomina auf-ianus ausmachen. ${ }^{101}$ Entsprechendes gilt für das restliche Imperium Romanum. Die einzige Ausnahme ist möglicherweise ein Nachweis aus Rom. Hier findet sich ein Mann namens Titianus Victorinus. Der miles classis Germaniciani stammte den Angaben seiner Grabinschrift zufolge aus Köln/CCAA [natio(ne) Agrippine(n)sis]. ${ }^{102}$ Es bleibt jedoch fraglich, ob der Mann lediglich zwei einfache Namen trug oder Titianus als ein Pseudogentiliz aufzufassen ist. ${ }^{103}$ Auf jeden Fall darf man vermuten, dass sein Name in Niedergermanien sicherlich anders interpretiert wurde als in Rom, da Pseudogentilnomina auf-ianus in Rom kaum bekannt waren und ein italisches Gentiliz Titianus nicht existiert.

96 Die Lesung des Namens bleibt unsicher. So wird der Name der Dedikantin in der EDH (Nr.HD040875) mit Quinciana Paterna wiedergegeben. In der EDCS (Nr.04900744) lautet die Lesung Ovinciana Paterna. SPICKERMANN 1994, 238. Nr.15, 245. Anm.101 entziffert dagegen das Gentiliz [Q] uincian[i]a (,Der Name ist offenbar eine falsche Bildung aus Quintus.“).

${ }^{97}$ Im CIL lautet die Lesung SABINIANVS. LEHNER 1918, 93-94. Nr.194 und ALFÖLDY 1968, 18. Nr.1 lesen SABINIANIVS mit einem hochgestellten I über dem V. Die Lesung findet sich u.a. auch bei SPICKERMANN 2008, 119. Nr.4 sowie in der EDCS (Nr.11100169) und in der EDH (Nr.HD077537). Bei dem vermeintlichen I, einem etwas schrägen Strich ohne Serifen, der nicht mittig über dem V steht, handelt es sich jedoch sicherlich nur um eine Verletzung des Steins. Zur Lesung des Namens s. KAKOSCHKE 2006, 341. GN 1077-4 (mit weiteren Hinweisen).

${ }^{98}$ Der Name des Verstorbenen wird im Genitiv genannt: T SIMPLICIANI ATTARACHI. B. und H. GALSTERER gaben den Namen im Nominativ zunächst mit Simplicianius [GALSTERER 1975, 83. Nr.356, 123 (Index)], dann mit Simplicianus wieder (IKöln ${ }^{2}$ 419. Nr.530). Beide Lesungen sind möglich. S. ferner Solin/SALOMIES ${ }^{2}$ 1994, 172 (Simplicianius), KAKOSCHKE 2006, 373. GN 1190 (Simplicianius), EDH Nr.HD076194 (Simplicianus).

${ }^{99}$ Zum miles classis Germaniciani s. weiter unten im Text.

100 2. N. 73 . Nr.78 (= EDH Nr.HD023928). Zur vermuteten Herkunft der Frau s. SPICKERMANN 1994, 303, KAKOSCHKE 2002, 297-298. Nr.2.79.

${ }^{101}$ Nicht berücksichtigt wurden zwei unsichere Belege aus der Gallia Lugdunensis: In Lyon/Lugdunum findet sich ein Mann namens Fortianus Paternus. Zu Fortianus s. Solin/SAlomIes ${ }^{2}$ 1994, 81 („GENTILICIUM?“), 277. Das OPEL hat den Namen nicht aufgenommen. - Aus derselben Stadt ist eine Frau namens Pantiana $Q$. f. Macrina durch eine nur literarisch bezeugte Grabinschrift bekannt (CIL XIII 2227). Laut den Angaben im CIL ist die Inschrift „,suspectus“. Zum Namen s. auch Solin/SAlOMIES² 1994, 137, 277 [,Pantianus(?)“].

${ }^{102}$ AE 2006, 394.

${ }^{103}$ Eine gewisse Unsicherheit hinsichtlich der Einordung des Namens Titianus gesteht auch die Ersteditorin der Inschrift ein; siehe M. G. GRANINO CECERE, ZPE 157 (2006), 241-242. 
Zusammenfassend kann man somit festhalten, dass Pseudogentilnomina auf -ianus wohl im ubischen Siedlungsgebiet „erfunden“ wurden. Alle datierbaren Belege der Namen stammen, wie auch die Nachweise für die gängigeren Pseudogentilnomina auf-ius und-inius (sowie-ianius) aus dem 2. und 3. Jahrhundert n. Chr. Die bemerkenswerte Bildungsweise eines Pseudogentilnomens fand im Gegensatz zu den konkurrierenden Bildungsweisen auf -ius, -inius und-ianius keine weitere Verbreitung in den keltisch geprägten Nachbarprovinzen und blieb ein Charakteristikum des ubisch geprägten Hinterlandes der CCAA. Ebenso wie die -ius- oder -iniusBildungen sind die -ianus-Namen ein Produkt der Romanisation einheimischer Bevölkerungsgruppen. Das beschriebene Phänomen ist sicherlich der Ausdruck einer romanisierten einheimischen Gesellschaft, die einen flexiblen und innovativen Umgang mit den eigenen Namenstrukturen pflegte, indem sie die eigenen Namenstraditionen dem römischen Namensystem der äußeren Form nach anpasste.

Die Pseudogentilnomina auf -ianus reihen sich damit ein in eine Kette weiterer epigraphischer bzw. onomastischer Besonderheiten aus dem Gebiet der CCAA. Genannt seien hier z.B. der Gebrauch der besonders beliebten -iniusNamen ${ }^{104}$ und der cognominalen Filiation ${ }^{105}$ sowie die Verwendung der Formel ex imperio ipsarum bzw. ipsius ${ }^{106}$ und eines besonderen Graphems in Form eines halben $\mathrm{H}$ in zahlreichen Matroneninschriften. ${ }^{107}$

\footnotetext{
104 Zu den Namen s. WEISGERBER 1968, 386-392.

105 G. ALFÖLDY, ES 4 (1967), 9-10 mit Karte 1 („Belege für die Angabe der Filiation durch ein Cognomen innerhalb der Tria Nomina in Niedergermanien“), A. KAKOSCHKE, GFA 18 (2015), 182, ders. FeRA 30 (2016), 11-13 (mit weiteren Literaturhinweisen).

${ }^{106}$ LIERTZ 2007, 844-845.

${ }^{107}$ A. KAKOSCHKE, FeRA 29 (2016), 60-70 (mit weiteren Literaturhinweisen).
} 


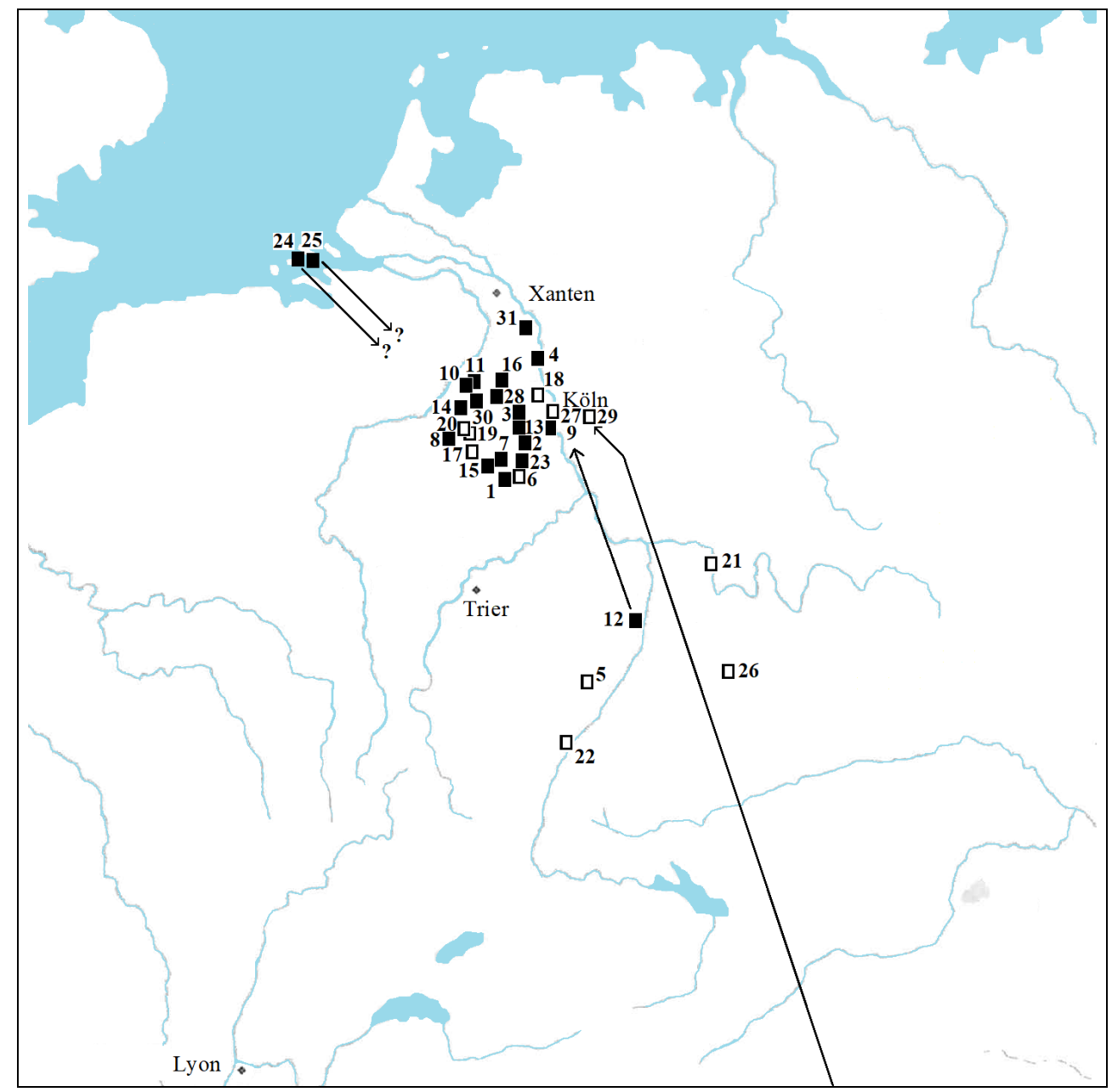

Abb.13: Verteilung der Pseudogentilnomina auf -ianus in den germanischen Provinzen und im angrenzenden Raum (Die angegebenen Nummern entsprechen denen, die in Tabelle 1 aufgelistet sind; $\mathbf{\square}=$ sicherer Beleg, $\square=$ unsicherer Beleg)

\section{Siglen}

1. N.

2. N.

3. N.

4. N.

$\mathrm{AE}$

H. FINKE, Neue Inschriften, BRGK 17 (1927), 1-107, 198-231.

H. Nesselhauf, Neue Inschriften aus dem römischen Germanien und den angrenzenden Gebieten, BRGK 27 (1937), 51-134.

H. Nesselhauf / H. LieB, Dritter Nachtrag zu CIL. XIII. Inschriften aus den germanischen Provinzen und dem Treverergebiet, BRGK 40 (1959), 120-229.

U. SCHILLINGER-HÄFELE, Vierter Nachtrag zu CIL XIII und zweiter Nachtrag zu FR. VOLLMER, Inscriptiones Baivariae Romanae. Inschriften aus dem deutschen Anteil der germanischen Provinzen und des Treverergebietes sowie Rätiens und Noricums, BRGK 58 (1977), 447-603.

L’Année Épigraphique, Paris.

http://www.fera-journal.eu 
ANRW

$\mathrm{BJb}$

$\mathrm{BNF}$

BRGK

CIL

EPRO

ES

GFA

IKöln ${ }^{2}$

KWDZ

OPEL I ${ }^{2}-\mathrm{IV}$

WZ

ZPE

AlBRECHT 1938

ALFÖLDY 1968

BAUCHHENß/TUTLIES 2006

BILLER 2010
Aufstieg und Niedergang der römischen Welt. Geschichte und Kultur Roms im Spiegel der neueren Forschung, Berlin-New York.

Bonner Jahrbuch, Bonn.

Beiträge zur Namenforschung, Heidelberg.

Bericht der Römisch-Germanischen Kommission, Mainz.

Corpus Inscriptionum Latinarum, Berlin 1863ff.

Études préliminaires aux religiones orientales dans l'empire romain, Leiden.

Epigraphische Studien, Köln-Bonn.

Göttinger Forum für Altertumswissenschaft, Göttingen. (www.gfa.gbv.de)

B. GALSTERER / H. GALSTERER, Die römischen Steininschriften aus Köln. IKöln ${ }^{2}$, Kölner Forschungen 10, Mainz 2010.

Korrespondenzblatt der Westdeutschen Zeitschrift, Trier.

Onomasticon Provinciarum Europae Latinarum $\mathrm{I}^{2}$-IV. Ex materia ab A. Mócsy, R. FELDMANN, E. MARTON et M. SZILÁGYI collecta, hrsg. von B. LŐRINCZ u.a., Budapest-Wien 1999-2005.

Westdeutsche Zeitschrift, Trier.

Zeitschrift für Papyrologie und Epigraphik, Bonn.

\section{Literaturverzeichnis}

Ch. Albrecht, Das Römerlager in Oberaden und das Uferkastell in Beckinghausen an der Lippe II, Veröffentlichungen aus dem Städt. Museum für Vor- und Frühgeschichte Dortmund 1. Bodenfunde, Münzen, Sigillaten und Inschriften nach Ergebnissen der Grabungen von A. BAUM, Dortmund 1938.

G. ALFÖLDY, Epigraphisches aus dem Rheinland III, ES 5 (1968), 1-98.

G. BAUCHHENß / P. TutLIES, Warum zweimal? Ein Trachytsockel aus dem Rotbachtal, Archäologie im Rheinland 2006, 134-137.

F. BILLER, Kultische Zentren und Matronenverehrung in der südlichen Germania inferior, Osnabrücker Forschungen $\mathrm{zu}$ Altertum und Antike-Rezeption 13, Rahden/Westf. 2010.

http://www.fera-journal.eu 
BROELMANN 1608

BYVANCK 1935

CAGNAT $^{4} 1914$

CALDELli 2018

CLAuSS 1976

DELAMARRE 2007

DERKS 2004

FORNI 1985

GALSTERER 1975

HAUG/SIXT ${ }^{2} 1914$

HONS 1826

KAJANTO 1965

KAKOSCHKE 2002

KAKOSCHKE 2006
St. BroelmanN, Epideigma sive specimen historiae veteris omnis et purae, florentis, atque amplae civitatis Ubiorum, et eorum ad Rhenum Agrippinensis oppidi, quod post Colonia Claudia Augusta Agrippinensis, Köln 1608.

A. W. ByVAnCK, Excerpta Romana. De bronnen der Romeinsche geschiedenis van Nederland. Tweede Deel. Tweede Afdeeling: Inscripties, 's-Gravenhage 1935.

R. Cagnat, Cours d'épigraphie Latine. 4. Aufl., Paris 1914.

M. L. CALDELLI, Epigrafia ostiense dopo il CIL. 2000 iscrizioni funerarie, Venedig 2018.

M. Clauss, Neue Inschriften im Rheinischen Landesmuseum Bonn, ES 11 (1976), 1-39.

X. Delamarre, Nomina Celtica antiqua selecta inscriptionum (Noms de personnes celtiques dans l'épigraphie classique), Paris 2007.

T. DERKS, Beelden en zelfbeelden van Bataven: de epigrafische bronnen, in: De Bataven. Verhalen van een verdwenen volk, hrsg. von L. SwINKELS, NijmegenAmsterdam 2004, 38-69.

G. FORNI, Le tribù romane III,1. Le pseudo-tribú, Rom 1985.

B. GALSTERER / H. GALSTERER, Die römischen Steininschriften aus Köln, Wissenschaftliche Kataloge des Römisch-Germanischen Museums 2, Köln 1975.

F. HAUG / G. SIXT, Die römischen Inschriften und Bildwerke Württembergs. Zweite, ergänzte und erweiterte Aufl., Stuttgart 1914.

Th. Hons, Vorläufige Mittheilungen über die MineralQuelle zu Heilstein unweit Aachen, Aachen 1826.

I. Kajanto, The Latin Cognomina, Societas Scientiarum Fennica. Commentationes Humanarum Litterarum 36.2, Helsinki-Helsingfors.

A. KAKOSCHKe, Ortsfremde in den römischen Provinzen Germania inferior und Germania superior. Eine Untersuchung zur Mobilität in den germanischen Provinzen anhand der Inschriften des 1. bis 3. Jahrhunderts n. Chr., Osnabrücker Forschungen zu Altertum und Antike-Rezeption 5, Möhnesee 2002.

A. KaKoschKe, Die Personennamen in den zwei germanischen Provinzen. Ein Katalog. Bd.1: Gentilnomina ABILIUS-VOLUSIUS, Rahden/Westf. 2006. 
KAKOSCHKE 2007

KAKOSCHKE 2008

LEHNER 1918

LIERTZ 2007

RAEPSAET-CHARLIER 2006

RAEPSAET-CHARLIER 2011

REUTER/SCHOLZ 2004

RICHIER 2004

RIESE 1914

RÜGER 1983
A. KakoschKe, Die Personennamen in den zwei germanischen Provinzen. Ein Katalog. Bd.2,1: Cognomina ABAIUS-LYSIAS, Rahden/Westf. 2007.

A. KAKoschKe, Die Personennamen in den zwei germanischen Provinzen. Ein Katalog. Bd.2,2: Cognomina MACCAUS-ZYASCELIS, Rahden/Westf. 2008.

M. Kemkes / N. Willburger, Der Soldat und die Götter. Römische Religion am Limes, Schriften des Limesmuseums Aalen 56, Esslingen am Neckar 2004.

K. KÖRBER, Inschriften (römische, griechische, mittelalterliche (auch Runen-)Inschriften) des Mainzer Museums. Dritter Nachtrag zum Becker`schen Katalog, Mainz 1900.

H. LeHNER, Die antiken Steindenkmäler des Provinzialmuseums in Bonn, Veröffentlichungen des Provinzialmuseums in Bonn 9, Bonn 1918.

U.-M. LIERTZ, Importierte und indigene Göttinnen im Nordwesten des Imperiums, in: International Congress of Greek and Latin Epigraphy. XII Congressus Internationalis Epigraphicae Graecae et Latinae. Barcelona. 3-8 Septembris 2002, Barcelona 2007, 841-846.

M.-TH. RAEPSAET-CHARLIER, Les dévots dans les lieux de culte de Germanie supérieure et la géographie sacrée de la province, in: M. DONDIN-PAYRE / M.-TH. RAEPSAET-CHARLIER (Hrsg.), Sanctuaires, pratiques cultuelles et territoires civiques dans l'Occident romain, Bruxelles 2006, 347-436.

M.-TH. RAEPSAET-CHARLIER, Les noms germaniques: adaptation et latinisation de l'onomastique en Gaule Belgique et Germanie inférieure, in: M. DONDIN-PAYRE (Hrsg.), Les noms de personnes dans l'Empire romain, Paris 2011, 203-234.

M. REUTER u. M. SchOlZ (Hrsg.), Geritzt und entziffert. Schriftzeugnisse der römischen Informationsgesellschaft, Schriften des Limesmuseums Aalen 57, Esslingen am Neckar 2004.

O. RICHIER, Centuriones ad Rhenum. Les centurions legionnaires des armées romaines du Rhin, Gallia Romana 6, Paris 2004.

A. RIESE, Das rheinische Germanien in den antiken Inschriften, Leipzig-Berlin 1914.

CH. B. RÜGER, Römische Inschriftenfunde aus dem Rheinland 1978-1982 (mit einem Beitrag von B. BEYER), ES 13 (1983), 111-166.

http://www.fera-journal.eu 
SCHÖPFLIN 1751

SCHULZE 1904

SCHWERTHEIM 1974

SELZER 1988

SOLIN/SALOMIES ${ }^{2} 1994$

SPICKERMANN 1994

SPICKERMANN 2003

SPICKERMANN 2008

STOLLWERCK 1877

WHATMOUGH 1970

WEISGERBER 1968

WILTHEIM 1842
J. D. SchöPflin, Alsatia illustrata I. Celtica Romana Francica, Colmar 1751.

W. Schulze, Zur Geschichte lateinischer Eigennamen, Abhdlg. der Königl. Ges. der Wiss. zu Göttingen. Phil.Hist. K1. N. F. 5, Göttingen 1904 (Nachdr. Berlin-Zürich-Dublin 1966).

E. Schwertheim, Die Denkmäler orientalischer Gottheiten im römischen Deutschland, EPRO 40, Leiden 1974.

W. SElzer, Römische Steindenkmäler. Mainz in römischer Zeit. Katalog zur Sammlung in der Steinhalle von W. SELZER unter Mitarbeit von K.-V. DECKER und A. Do PAÇO, Mainz 1988.

H. Solin / O. SAlomies, Repertorium nominum gentilium et cognominum Latinorum. 2. Aufl. Editio nova addendis corrigendisque augmentata, Alpha Omega. Reihe A. Lexika - Indizes - Konkordanzen zur klassischen Philologie 80, Hildesheim-Zürich-New York 1994.

W. SPICKERMANN, „Mulieres ex Voto“. Untersuchungen zur Götterverehrung von Frauen im römischen Gallien, Germanien und Rätien (1.-3. Jahrhundert n. Chr.), Bochumer historische Studien. Alte Geschichte 12, Bochum 1994.

W. SPICKERMANN, Germania superior. Religionsgeschichte des römischen Germanien I, Religion der Römischen Provinzen 2, Tübingen 2003.

W. SPICKERMANN, Germania inferior. Religionsgeschichte des römischen Germanien II, Religion der Römischen Provinzen 3, Tübingen 2008.

F. STOLLWERCK, Die celtubisch-römische Niederlassung Gelduba zwischen Novesium und Asciburgium, Uerdingen 1877.

J. Whatmough, The dialects of ancient Gaul. (Mikrofilm) Cambridge/Massachusetts 1949ff. (= Nachdr. Cambridge/Massachusetts 1970).

J. L. WeISGERBER, Die Namen der Ubier, Wissenschaftliche Abhandlungen der Arbeitsgemeinschaft für Forschung des Landes Nordrhein-Westfalen 34, Köln-Opladen 1968.

A. WiLtheim, Luciliburgensia sive Luxemburgum Romanum, Luxemburg 1842. 


\section{Elektronische Datenbanken}

EDCS

Epigraphik-Datenbank Clauss/Slaby (Frankfurt), Leitung: M. ClaUSS u.a. (Johann Wolfgang Goethe-Universität Frankfurt am Main / Katholische Universität Eichstätt-Ingolstadt / Universität Zürich), Stand: 04.03.2019 (www.manfredclauss.de).

$\mathrm{EDH}$

Epigraphische Datenbank Heidelberg, Leitung: $\mathrm{CH}$. WITSCHEL (Ruprecht-Karls-Universität Heidelberg), Stand: 04.03.2019 (www.uni-heidelberg.de/institute/ sonst/adw/edh).

EDR

Epigraphic Database Roma, Leitung: S. PANCIERA, S. ORLANDI (Università di Roma - La Sapienza), Stand: 04.03.2019 (www.edr-edr.it).

LUPA

UBI ERAT LUPA, Bilddatenbank zu antiken Steindenkmälern (Universität Salzburg), Leitung: F. und O. HARL (Universität Wien), Stand: 04.03.2019 (www.ubi-eratlupa.org).

\section{Kontakt zum Autor:}

Dr. Andreas Kakoschke

Nelly-Sachs-Weg 1

D-49191 Belm

E-Mail: andreaskakoschke@,hotmail.de 